\title{
Introduction du traitement médical d'urgence ainsi que les services et conseils ultérieurs en planification familiale pour les femmes qui souffrent de complications suite à un avortement au Burkina Faso
}

Population Council

JPIEGO

Follow this and additional works at: https://knowledgecommons.popcouncil.org/departments_sbsr-rh How does access to this work benefit you? Let us know!

\section{Recommended Citation}

"Introduction du traitement médical d'urgence ainsi que les services et conseils ultérieurs en planification familiale pour les femmes qui souffrent de complications suite à un avortement au Burkina Faso," Final Report. Nairobi: Population Council, Min Santé Burkina Faso, Cellule Recherche Santé Repro., Maternité Ctre Hosp Nat Yalagao Ouédraogo, and JHPIEGO, 1998. 


\section{Ministère de la Santé du Burkina Faso}

Direction de la Santé de la Famille

Cellule de Recherche en Santé de la Reproduction (CRESAR)
Maternité du

Centre Hospitalier

National

Yalagado Ouédraogo

Introduction du traitement médical d'urgence ainsi que les services et conseils ultérieurs en planification familiale pour les femmes qui souffrent de complications suite à un avortement au Burkina Faso

Population Council Projet RO/AT en Afrique II

JHPIEGO

Décembre 1998 


\section{Ministère de la Santé du Burkina Faso \\ Direction de la Santé de la Famille}

Cellule de Recherche en Santé de la Reproduction (CRESAR)

\author{
Maternité du \\ Centre Hospitalier \\ National \\ Yalagado Ouédraogo
}

Introduction du traitement médical d'urgence ainsi que les services et conseils ultérieurs en planification familiale pour les femmes qui souffrent de complications suite à un avortement au Burkina Faso

\section{Population Council Projet RO/AT en Afrique II}

\section{JHPIEGO}

Cette étude a été menée dans le cadre du deuxième Projet de Recherches Opérationnelles et d'Assistance Technique (RO/AT) en Afrique du Population Council et les fonds centraux de l'USAID alloués a JHPIEGO. Le Projet RO/AT II est financé par "I'Agence des Etats Unis pour le Développement Internationale" (USAID), Bureau de la Population, Contrat $\mathrm{N}^{\circ} \mathrm{CCP}-3030-\mathrm{C}-00-$ 3008-00, Stratégies pour améliorer la prestation des services de Planification Familiale.

Le soutien financier de JHPIEGO vient de son contrat de coopération No. CCP-A-00-93-00020-00 avec l'Agence des Etats Unis pour le Développement International.

Les opinions exprimées dans ce document sont celles de ses auteurs et ne reflètent pas nécessairement les vues de l'USAID. 


\section{Personnes ayant participé à l'étude}

CRESAR \& Maternité du Centre Hospitalier National Yalagado Ouédraogo et Centre Hospitalier Sanou Sourou de Bobo-Dioulasso

$\underline{\text { CHU Ouagadougou }}$

Professeur Bibiane Koné

Dr. Jean Lankoandé

Dr. Blandine Tiéba

Dr. Fatoumata Traoré

Dr. Michel Akotionga

Dr. Charlemagne Ouédraogo

Dr. Max Jonas Gué

$\underline{\text { CHU Bobo-Dioulasso }}$

Dr. André Jules Bazié

Dr. Blami Dao

Ministère de la Santé Publique - Direction de la Santé de la Famille

Dr. Paul Stanislas Nebié

Dr. Ernest Dabiré

Ministère de la Santé Publique - Direction des Etudes et de la Planification

Dr. Alain Zoubga

Population Council - Projet RO/AT en Afrique

Dr. Placide Tapsoba

Dr. Inoussa Kaboré

JHPIEGO

Ms. Anita Ghosh

Ms. Kathy Jesencky

Dr. Shasha Wilbrod 


\section{Table des Matières}

Liste des abréviations

Page

Liste des graphiques

i

Liste des tableaux

Executive summary

iii

iv

I. INTRODUCTION 1

II. JUSTIFICATION DE L'ETUDE 2

III. OBJECTIFS ET HYPOTHESES 2

3.1.Objectif à long terme 2

3.2. Objectifs à court terme 2

3.3.Hypothèses de l'étude 3

IV. APPROCHE METHOLOGIQUE 4

4.1. Schéma de l'étude 4

4.2. Cadre de l'étude $\quad 4$

4.3. Les parties impliquées dans la recherche 4

4.4. Type d'échantillonnage $\quad 5$

4.5. Définitions opérationnelles 5

4.6. Collecte et analyse des données $\quad 6$

4.6.1. Collecte des données par interviews auprès 6

4.6.2.Saisie et analyse des données $\quad 6$

4.7. Aspects éthiques 6

V. DESCRIPTION DE L'INTERVENTION 7

5.1. Les activités préparatoires $\quad 7$

5.1.1. Recensement des besoins en formation. $\quad 7$

5.1.2. Développement de politiques et standards

en matière de soins post abortum $\quad 7$

5.2. Formation des prestataires de soins $\quad 7$

5.2.1. Formation en prévention des infections $\quad 7$

5.2.2. Mise à jour en technologie contraceptive 8

5.2.3. Formation en $A M I U$

5.2.3. Organisation d'une journée d'information et d'orientation 8

5.3. Intégration du counseling en PF dans la prise

en charge des patientes 
$\begin{array}{ll}\text { VI. LES RESULTATS } & 9\end{array}$

6.1. Répartition de la population enquêtée $\quad 9$

6.2. Profil de la population enquêtée $\quad 10$

6.2.1. Caractéristiques socio-démographiques des patientes 10

6.2.2. Connaissances des patientes sur la péiode de la fécondité 10

6.3. Données cliniques 13

6.3.1. Type d'avortement 13

6.3.2. Motif de consultation 14

6.4. Prise en charge des Patientes 14

6.4.1. Technique d'évacuation utérine 14

6.4.2. Procédure de contrôle de la douleur $\quad 15$

6.4.3. Perception de la douleur par les patientes $\quad 15$

6.4.4. Informations reçues par les patientes 16

6.5. Durée d'hospitalisation $\quad 16$

6.6. Satisfaction des patientes $\quad 17$

6.7. Planification familiale chez les patientes 18

6.7.1. Connaissance de la planification familiale 18

6.7.2. Intention d'utiliser la planification familiale $\quad 19$

6.7.3. Acceptation de la planification familiale 21

6.7.4. Qualité des prestations 22

6.7.5. Implication du conjoint à la planification familiale 23

6.8. Les prestataires des services $\quad 25$

6.8.1. Caractéristiques socio-démographiques et professionnelles 25

6.8.2. Formation en soins post abortum 25

6.8.3. Formation en planification familiale après avortement 26

6.8.4. Connaissance par les prestataires de la période de fécondité $\quad 27$

6.9. Les coûts des prestations de services $\quad 26$

6.9.1. Coûts supportés par la patiente 26

6.9.1.1. Coûts des soins 26

6.9.1.2. Les coûts de l'hospitalisation $\quad 27$

6.9.2. Coûts supportés par l'institution 28

6.10. La qualité des soins : opinions des patientes 29

6.11. Connaissance des dispositions juridiques et règlementaires / opinions sur la législation en matière d'avortement 30

$\begin{array}{ll}\text { VII. CONCLUSION } & 31\end{array}$

VIII. RECOMMANDATIONS 32

8.1. Par rapport au programme SPA 32

8.2. Par rapport aux prestations 32

IX. BIBLIOGRAPHIE 33 


\section{Liste des abréviations}

AMIU

CHN-SS

CHN-YO

CIPD

CRESAR

$\mathrm{D} \& \mathrm{C}$

DIU

EDS

DSF

FNUAP

IVG

JH PIEGO

Aspiration Manuelle Intra Utérine

Centre Hospitalier National Sanou Sourou

Centre Hospitalier National Yalgado Ouédraogo

Conférence Internationale sur la Population et le

Développement

Cellule de Recherche en Santé de la Reproduction

Dilatation et Curetage

Dispositif Intra Utérin

Enquête Démographique et de Santé

Direction de la Santé de la Famille

Fonds des Nations Unies pour la Population

Interruption Volontaire de Grossesse

Programme de John Hopkins pour l'Education et la Formation Internationales en Matière de Santé de la Reproduction

MS

MTC

OMS

ONG

PA

$\mathrm{PF}$

PI

$\mathrm{RO} / \mathrm{AT}$

SPA

SMI

SR

USAID

Ministère de la Santé

Mise à jour en Technologie Contraceptive

Organisation Mondiale de la Santé.

Organisation Non Gouvernementale

Post Abortum

Planification Familiale

Prévention des Infections

Recherche Opérationnelle/Assistance Technique

Soins Post Avortement

Santé Maternelle et Infantile

Santé de la Reproduction

Agence des Etats Unis pour le Développement International 


\section{Liste des graphiques}

Page

Fig 1 : Répartition des patientes selon la tranche d'âge ...................................... 10

Fig 2 : Niveau de connaissance des patientes .................................................... 13

Fig 3 : Répartition selon le type d'avortement .................................................. 13

Fig 4 : Informations données aux patientes ................................................... 15

Fig 5 : Durée moyenne d'hospitalisation selon la technique .............................. 17

Fig 6 : Durée d'hospitalisation en cas d'AMIU selon le site ................................ 17

Fig 7 : Tendances des intentions d'utiliser la PF par rapport au nombre d'enfants vivants par patiente ............................................. 21

Fig 8 : Type de méthode Contraceptive reçu

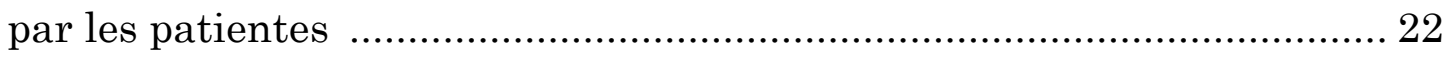

Fig 9 : Approbation de la femme et approbation

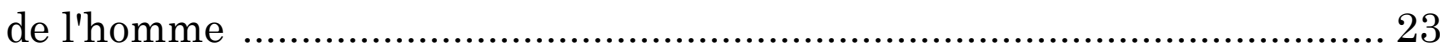

Fig 10 : Coûts moyens des dépenses supportées

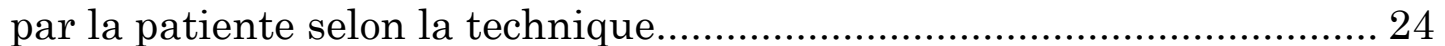

Fig 11 : Points de vue des patientes sur la loi sur l'avortement provoqué au Burkina Faso 


\section{Liste des tableaux}

Page

Tableau I : Répartition des patientes et les prestataires selon le site et la phase d'étude..................................................... 9

Tableau II : Répartition des patientes selon leur histoire reproductive .............. 12

Tableau III : Répartition des patientes selon la technique ................................. 14

Tableau IV : Niveau de satisfaction des patientes............................................. 18

Tableau V : Prestations de la PF / Counseling à l'intention

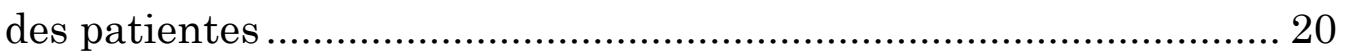

Tableau VI : Qualité des prestations en planification familiale

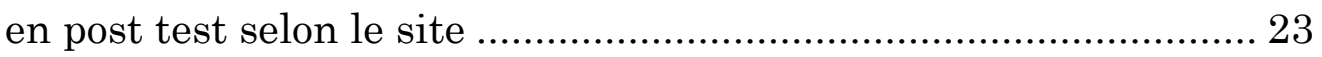

Tableau VII : Caractéristiques socio-démographiques du personnel.................... 25

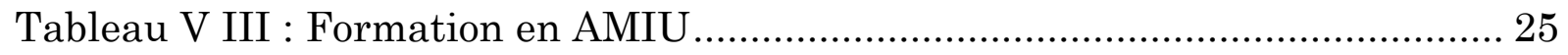

Tableau IX: Informations et prestations de services PF ................................ 26 


\section{Executive summary}

This operations research study examined the feasibility and acceptability of establishing post abortion care (PAC) services to improve clinical care and follow-up of post abortion patients in two pilot sites in Burkina Faso. A pre-test / post-test, no control group study design was utilized to measure the effects of an intervention that upgraded physicians' and midwives' clinical and interpersonal communication skills for the care of post abortion patients, including counseling and family planning.

The project represents the first effort to introduce PAC services in francophone West Africa. It was anticipated that the approach could be adapted for similar settings in the West Africa region. The study was implemented for the Family Health Directorate (DSF), Ministry of Health by the Burkina Faso Chapter of the Reproductive Health Research Network (CRESAR) in collaboration with the Population Council Africa Operations Research and Technical Assistance (OR/TA) Project and the JHPIEGO Corporation. In addition, assistance from the UNFPA and the Family Health and AIDS Prevention Project for West and Central Africa was solicited and provided at different stages during the project.

The study's pre- and post-test surveys utilized direct interviews with staff working in the maternity wards and with patients prior to their discharge. Costs related to the treatment for both patients and the hospitals were also collected. The training intervention, carried out by JHPIEGO, was comprised of a series of activities designed to strengthen the sites and prepare providers to offer PAC services :

1. Advocacy and consensus-building: needs assessment, preliminary meetings to gain consensus, development of policies and standards for PAC services and orientation meetings.

2. Training for PAC service delivery: training in use of recommended infection prevention practices, contraceptive technology update workshop, PAC training and follow-up visits.

Caring for the total needs of the patients was stressed as an important element of the training strategy. The non-MVA related training focused on communicating with the patient and the importance of PAC family planning counseling. Training for nurses and other para-medical personnel was also provided. Participants learned how to counsel patients and manage uncomplicated cases as well as lifethreatening emergencies. An original protocol for the management of incomplete abortion was developed which included guidelines for the treatment of all abortion complications. Changes in the clinical management of post abortion patients were introduced through JHPIEGO supported site-based training for physicians and nurses in Burkina Faso and a training of four Ob/Gyn specialists in Korle Bu Teaching Hospital in Accra, Ghana.

A total of 786 patients were interviewed during the study. During the pre-test 330 patients were interviewed; 456 during the post-test in both sites. The pre- and post-test samples of patients did not exhibit any significant differences in terms of their socio-demographic characteristics. 
Approximately 53 percent of the post abortion patients reported a previous history of using contraceptives including 7 percent of the post abortion patients who reported using a modern contraceptive at the time of conception.

Nine percent of physicians (10), during the pre-test interview, reported having received training in the use of MVA, even though none of them were using MVA in the hospitals. At the time of the post-test, around half of the physicians at the study sites were trained. The introduction of PAC has largely contributed to increased accessibility and availability of family planning services in the two maternity sites. During the pre-test 45 percent of health providers reported that they were trained in family planning while this number increased to 71 percent in the post-test as a result of the training in contraceptive technology update.

Knowledge about return to fertility of post abortion patients was persistently deficient among providers during the pre-test survey (29 percent). During the post-test, about 70 percent of providers demonstrated a great improvement in the knowledge of risk of PAC patients in conceiving another pregnancy during unprotected sex as early as the two weeks following an abortion. This has had positive implications in the prescription of PAC family planning for patients.

The study had a substantial effect on changing surgical procedures for ensuring the safe and complete evacuation of uterine contents. Dilatation and Curetage (D\&C) and digital curage were the main techniques used in almost 100 percent of cases (60 and 40 percent respectively) prior to the study. The average number of cases treated with D\&C after the introduction of MVA at the maternities, dropped to 1.8 percent, with digital curage to 1.1 percent, while use of MVA went up to 97.1 percent.

The study intervention also introduced the use of local anesthesia with preoperative sedation and/or analgesics as indicated. Prior to the study general anesthesia was routinely used in almost all cases. After the introduction of MVA, 98 percent of patients treated with the new technique were prepared with psychological support through "verbal anesthesia", accompanied with local anesthesia for about 27 percent of cases and 6 percent received a post-treatment analgesic for pain control.

The records showed that the introduction of the new approach to treating post abortion patients is associated with a statistically significant shorter hospital stay resulting in savings in hospital resources as well as financial savings to the patients. The study's results also strongly suggest a positive impact on interpersonal communication between health providers and post abortion patients. The exchange of information became substantially more frequent after the intervention.

Family planning was not routinely provided prior to the study. Results from the pre-test survey show that only 30 percent of post abortion patients were reported having been counseled about family planning issues. During the post-test survey, nearly 95 percent of the patients had these topics discussed with them by a 
provider. The study had a significantly positive effect on the post abortion patients intention to begin using a contraceptive method. The proportion of patients with the intention to begin use of a contraceptive method increased from 64 percent at the pre-test to 82 percent at the post-test. The use of the method by post abortion patients went from 57 percent to 84 percent after the intervention.

CRESAR has made a bold first step in pioneering PAC services in Burkina Faso and in the region. Results from this study demonstrate that the introduction of a more comprehensive approach to PAC services, including the use of MVA, family planning counseling and services and referral to other reproductive health services, offers significant potential benefits for women, service providers and the health care system. Now the challenge is to consolidate the experience gained from the pilot study and expand the program while ensuring the quality of the services. Assistance to other countries in the francophone region such as Senegal, GuineaConakry and others is being planned for the decentralization of services delivery. 


\section{INTRODUCTION}

La question de l'avortement au Burkina Faso est d'une grande importance dans le domaine de la santé de la reproduction. Compte tenu de la délicatesse de ce problème de santé, les médecins se focalisaient sur une de ses dimensions à savoir, le traitement de ses aspects physiologiques. Les effets pervers d'ordre psychosocial ainsi que la nécessité du recours à la planification familiale comme mesure préventive de futures grossesses non désirées étaient insuffisamment pris en compte.

Auparavant, toutes les complications d'avortement au Burkina Faso étaient prises en charge par la technique de dilatation et curetage (D\&C). Les patientes étaient admises à l'hôpital et le plus souvent devaient attendre plusieurs heures avant de recevoir une évacuation utérine dans la principale salle d'opération. Le procédé était fait sous calmant puissant ou sous anesthésie générale, ce qui supposait que la malade devait normalement passer une nuit hospitalisée. Une étude menée dans deux hôpitaux de district au Kenya a montré que la durée moyenne du séjour des patientes à l'hôpital après un curetage à fond était de deux jours (40,9 heures) dans l'un des hôpitaux et plus de quatre jours dans l'autre (100,7 heures) (Johnson et al).

Afin de développer des liens étroits entre le traitement des complications de l'avortement et la prestation des services de planification familiale, le concept des soins post avortement (SPA) a été défini sur le plan international. Ce concept renferme trois éléments clés :

- Services de traitement d'urgence des complications de l'avortement spontané ou de l'avortement clandestin,

- $\quad$ Services de planification familiale et de counseling après avortement,

- Liens entre les services de traitement d'urgence de l'avortement et l'ensemble des soins complets de la santé de la reproduction (Greenslade et al).

La présente étude a mis l'accent essentiellement sur les deux premiers éléments des soins post-avortement à savoir le traitement d'urgence des complications et la planification familiale post-avortement soutenus par une recherche. 


\section{JUSTIFICATION DE L'ETUDE}

Chaque année de 40 à 60 millions de femmes à travers le monde cherchent à interrompre une grossesse non désirée. L'OMS et l'Institut Alan Guttmacher estiment qu'à nos jours les décès par suite d'avortement représentent $15 \%$ de la mortalité maternelle y compris les ajustements des cas non reportés.

Une femme sur 5 désireuse d'éviter une grossesse, n'utilise aucune forme de contraception moderne. En Afrique, en moyenne $17 \%$ de femmes mariés en âge de procréer utilisent la contraception. Au Burkina Faso, la couverture contraceptive est de $8,32 \%$. L'avortement provoqué trahit un échec de l'accessibilité et surtout de l'utilisation de la planification familiale.

Un décès sur 8 lié à la grossesse est dû à l'avortement à risque. Au Burkina Faso, 4 à $6 \%$ des décès maternels hospitaliers sont liés aux complications d'avortements provoqués.

Beaucoup de femmes qui survivent à cette pratique doivent faire face à un certain nombre de complications dont les infections, l'hémorragie, la perforation utérine, le traumatisme cervical, occasionnant à long terme la stérilité ainsi que des dégâts psychologiques (Coeytaux, 1990, McLaurin et al. 1991). De plus, les avortements provoqués, faits dans de mauvaises conditions d'asepsie entraînent de nombreux frais au plan économique, le plus évident étant l'augmentation de l'utilisation de ressources sanitaires rares pour le traitement en urgence de ces complications.

Le Programme d'Action de la Conférence Internationale sur la Population et le Développement qui s'est tenue au Caire en 1994, définit les soins après avortement comme une activité prioritaire.

C'est dans ce contexte que la Cellule de Recherche en Santé de la Reproduction (CRESAR) en collaboration avec la DSF, le Projet RO/AT du Population Council et JHPIEGO a développé ce projet pour améliorer les soins après avortement des clientes dans deux hôpitaux au Burkina Faso.

\section{OBJECTIFS ET HYPOTHESES}

\subsection{Objectif à long terme}

Améliorer la qualité des soins post abortum et développer des liens étroits entre le traitement des complications de l'avortement et les prestations de planification familiale, afin de diminuer le risque de morbidité et de mortalité maternelle au Burkina Faso.

\subsection{Objectifs à court terme}

a. Améliorer les compétences cliniques des spécialistes en gynécologie et obstétrique dans le traitement des avortements 
incomplets, provoqués ou spontanés : utilisation des procédures de contrôle de l'infection et de la douleur;

b. Améliorer le système de conseils en planification familiale ainsi que les compétences de communication interpersonnelle du personnel médical chargés de fournir des services aux femmes qui ont reçu des soins pour une fausse couche inachevée ou un avortement provoqué.

c. Améliorer la connaissance et l'utilisation des services de planification familiale chez les femmes qui suivent un traitement après une fausse couche ou un avortement provoqué.

d. Implanter deux sites au Burkina Faso pouvant servir de modèles de sites de formation dans le pays pour les conseils et les soins après avortement.

e. Décrire et comparer le coût, la faisabilité et l'introduction de services de l'aspiration manuel intra-uterine (AMIU) pendant la phase post-intervention et les coûts des services de dilatation et curetage pendant la phase pré-intervention.

\subsection{Hypothèses de l'étude}

i) La formation des prestataires de gynécologie et d'obstétrique dans les compétences cliniques pour la prestation des services d'AMIU aidera à améliorer leur connaissance technique de traitement des complications de l'avortement, d'utilisation des procédures de contrôle de l'infection et des procédures plus appropriées de contrôle de la douleur.

ii) La prestation des services d'AMIU aux femmes qui se présentent avec un avortement incomplet réduira leur temps d'hospitalisation, aboutira à une plus grande satisfaction et à une réduction de la perception de la douleur chez la patiente par rapport à l'utilisation des procédures de curetage.

iii) La mise en place d'un système de conseils en planification familiale pour des femmes sous soins médicaux suite à des avortements provoqués ou à une fausse couche aura un impact positif sur leur connaissance de la planification familiale ainsi que sur leur intention de l'utiliser.

iv) Il y aura des différences importantes au niveau des coûts : l'introduction des services d'AMIU aura un impact dans la réduction des coûts des services par rapport à l'utilisation de la technique de la dilatation et curetage. 


\section{APPROCHE METHOLOGIQUE}

\subsection{Schéma de l'étude}

L'étude a utilisé un modèle pré-intervention / post-intervention pour examiner les hypothèses entre la prestation des services d'AMIU et les services $\mathrm{PF}$ post avortement. Il n'a pas été nécessaire d'avoir un site témoin puisque les données de la pré-intervention seront suffisantes pour les besoins de la comparaison. Les données de la post-intervention ont été collectées 8 semaines après l'introduction de la nouvelle approche de la prise en charge des patientes. Les mesures pré-intervention et la post-intervention ont été faites pendant une période de 4 mois. L'étude peut être représentée par le diagramme suivant :

Expérimental
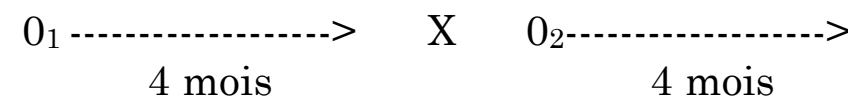

Où :

$0_{1}, 0_{2}=\quad$ Interviews réalisées à partir des questionnaires auprès des patientes souffrant de complications post-abortum pour collecter les données sur :

- Les caractéristiques socio-démographiques;

- Les raisons de l'avortement / fausse couche;

- La satisfaction par rapport aux prestations de soins;

- La perception de la douleur au cours de la procédure;

- La connaissance de la PF et l'intention de l'utiliser

- Le rôle du partenaire.

$\mathrm{X}=\quad$ Intervention : Mise en application de la nouvelle approche de prise en charge des patientes comprenant ;

- La formation en AMIU, PF, PI, et en counseling

- Réorganisation des services (équipement, introduction $\mathrm{PF})$.

\subsection{Cadre de l'étude}

L'étude s'est déroulée à Ouagadougou et à Bobo-Dioulasso dans les deux Centres Hospitaliers Nationaux Universitaires du Burkina Faso.

\subsection{Les parties impliquées dans la recherche}

La réalisation de l'étude a impliqué plusieurs institutions, chacune jouant un rôle complémentaire et unique en fonction de sa spécialité.

La CRESAR : La Cellule de Recherche en Santé de la Reproduction (CRESAR), membre du Réseau Africain de Recherche en Santé de la Reproduction a été crée en 1989 comme une organisation non gouvernementale (ONG) par le Gouvernement Burkinabé. Compte tenu de sa composition multidisciplinaire et de son expérience en matière de recherche en santé de la reproduction (SR), elle a été chargée de l'exécution de cette étude et de la dissémination de ses résultats. 
Direction de la Santé de la Famille (DSF): C'est la direction centrale assurant la coordination de l'ensemble des activités de SR au sein du Ministère de la Santé (MS) au Burkina Faso. A ce titre, elle assure la coordination de l'étude et est responsable de la dissémination et de l'utilisation des résultats de l'étude. Elle a participé à toutes les réunions de concertation et de coordination de l'étude

FNUAP : Le Fonds des Nations Unies pour la Population (FNUAP) a fourni une assistance financière à la CRESAR qui a permis la mise en place d'une unité de $\mathrm{PF}$ à la maternité du CHN-YO et l'acquisition de fongibles et d'équipements techniques pour les soins intensifs dans chacun des deux hôpitaux mais aussi d'améliorer les locaux du service de planification familiale.

JHPIEGO : JHPIEGO, le programme de Johns Hopkins pour l'Education et la Formation Internationales en Matière de Santé de la Reproduction est l'une des plus grandes institutions spécialisées dans la formation en prestation des services de santé de la reproduction. JHPIEGO a fourni les financements, l'appui technique et la logistique nécessaire pour l'exécution du volet intervention.

Population Council: Le Projet RO/AT du Population Council, a une longue expérience en matière de recherche en SR à travers le monde entier et particulièrement en Afrique. Le Projet RO/AT a fourni les financements, l'appui technique et la logistique nécessaire pour la gestion et l'exécution du volet recherche. Il a apporté son appui pour la dissémination et l'utilisation des résultats.

\subsection{Type d'échantillonnage}

L'échantillon de l'étude est constitué par l'ensemble des femmes en âge de procréer, admises dans les services de gynéco-obstétrique des Centres Hospitaliers Nationaux de Ouagadougou (site 1) et de Bobo Dioulasso (site 2) au cours des périodes allant du 20 avril 1997 au 20 septembre 1997 pour la première phase et du 21 janvier 1998 au 21 mai 1998 pour la seconde phase. Ont été inclues dans l'échantillon les patientes admises pour avortement incomplet spontané ou provoqué et dont l'âge gestationnel est inférieur ou égal à 15 semaines d'aménorrhée. Ont été exclues, les patientes présentant des complications médicales majeures (pelvipéritonite, perforation uro-génitale) et celle ayant refusées les interviews.

\subsection{Définitions opérationnelles.}

- L'avortement provoqué : l'avortement a été caractérisé de provoqué lorsqu'il a été confirmé par la patiente elle-même par un aveu.

- L'avortement provoqué suspect : Devant des signes cliniques de lésions génitales ou d'intoxication/infection pouvant faire évoquer une manœuvre abortive. 
- L'avortement était considéré comme spontané lorsque aucune des conditions précédentes n'était retrouvée.

- Utilisation passée d'une méthode contraceptive moderne : Nous avons utilisé ce terme pour indiquer l'utilisation passée d'au moins un mois avant l'épisode d'avortement d'un moyen contraceptif moderne par la patiente. La durée d'utilisation est au moins supérieure à un cycle (1 mois).

- Phase de pré-intervention : Période de 4 mois précédant l'introduction de la nouvelle approche de prise en charge des patientes et ayant servie pour la collecte des données de base.

- Phase de post-intervention : Période de 4 mois après l'introduction de la nouvelle approche de prise en charge des patientes et ayant servie pour la collecte des données.

\subsection{Collecte et analyse des données}

\subsubsection{Collecte des données par interviews auprès :}

des patientes : Les données ont été collectées à l'aide de questionnaire individuel auprès des patientes. Le moment et le lieu de ces interviews ont été coordonnés avec le personnel de la maternité chargé de la gestion de ces patientes. Des enquêtrices, au nombre de deux pour chaque site, ont été recrutées et formées pour la collecte des données.

Une évaluation des coûts des prestations selon la méthode développée par Ipas (M. Abernathy et al) et adaptée à notre contexte a permis la collecte des coûts relatifs à la prise en charge par la technique de D\&C pendant la phase préintervention et la prise en charge par la technique d'AMIU pendant la phase post-intervention.

des prestataires: Des interviews individuelles ont été faites auprès des prestataires des services médicaux pour évaluer leur connaissance des complications de l'avortement, et leur connaissance et attitude par rapport à la $\mathrm{PF}$ pour les patientes en post abortum. Ces interviews ont été menées auprès de tous les prestataires de chaque site.

\subsubsection{Saisie et analyse des données}

Les données de l'étude ont été saisies sur le logiciel d'analyse de données Epi-Info. Les procédés d'analyse se sont focalisés au départ sur les analyses univariées d'indicateurs. Les analyses bivariées et multivariées ont été utilisées pour déterminer l'importance statistique des différences dans les variables dépendantes clés, et ainsi tester les hypothèses énumérées ci-dessus.

\subsection{Aspects éthiques}

Le choix éclairé et la confidentialité sont essentiels dans une étude portant sur une question aussi sensible. Les enquêteurs ont été formés dans les procédures d'obtention du choix éclairé, et les principaux co-enquêteurs suivaient de près cet aspect de la collecte des données. Ils ont été précédemment impliqués 
dans les essais de l'introduction de l'implant Norplant ${ }^{\circledR}$ au Burkina Faso et de ce fait, sont habitués aux procédures du choix éclairé.

Des arrangements ont été faits avec le personnel hospitalier pour identifier un lieu pour l'interview où l'intimité totale était possible. Avant le début de l'interview, les patientes étaient informées du but de l'étude et il leur était demandé leur adhésion volontaire à l'étude. Elles étaient également informées que leur nom ne figurera pas sur le questionnaire qui sera archivé dans les bureaux de la CRESAR.

\section{DESCRIPTION DE L'INTERVENTION}

\subsection{Les activités préparatoires}

Avant l'introduction de la nouvelle procédure, des étapes préliminaires ont été nécessaires et utiles. Plusieurs réunions ont été tenues pour rechercher un consensus des acteurs clefs autour du projet, à savoir le MS et les responsables de la CRESAR.

\subsubsection{Recensement des besoins en formation.}

Cette activité conduite par l'équipe de JHPIEGO a permis d'identifier les besoins non seulement sur l'AMIU mais aussi sur les services connexes à l'offre d'un service de qualité tel que la prévention des infections, la circulation des clientes, la mise à jour en technologie contraceptive et en counseling. Cette évaluation des besoins s'est focalisé sur l'observation des prestataires de services dans leur milieu, sur l'interview du personnel et la revue des dossiers. A partir des résultats de ce recensement, des formations ont été menées en fonction des lacunes détectées.

\subsubsection{Développement de politiques et standards en matière de soins post abortum}

L'une des activités préparatoires la plus importante a été le développement d'un document de politiques et standards en matière de prise en charge des avortements. Le Ministère de la Santé, les responsables de CREASR et les prestataires ont pris activement part à cette activité. Le rôle du counseling avant, pendant et après le traitement a été reconnu comme une section importante de ce document.

\subsection{Formation des prestataires de soins}

\subsubsection{Formation en prévention des infections}

La prévention des infections est une nécessité préalable à l'offre de services adéquats et de qualité en matière de soins post abortum. Cette formation a concerné non seulement les responsables des unités des deux maternités mais tout le personnel impliqué dans le traitement et la gestion des équipements (gants, instruments, champs, etc.). Elle a été une opportunité pour introduire le concept de soins post-avortement aux prestataires. 


\subsubsection{Mise en jour en technologie contraceptive}

L'évaluation des besoins a révélé une lacune en matière de prestation des services de PF au niveau des prestataires. Pour cela, un atelier de six jours en technologie contraceptive/soins post abortum a été organisé pour le personnel des deux maternités.

\subsubsection{Formation en AMIU}

La formation sur la technique de l'AMIU a été assurée au personnel des services de gynécologie et obstétrique pour le traitement des avortements incomplets. Le diagnostic et le traitement des complications courantes, mineures ou mortelles, ont fait l'objet d'une mise à jour.

La formation initiale en counseling, s'est déroulée à Ouagadougou pour une quinzaine de prestataires suivie d'une autre en AMIU à Accra au Ghana pour une équipe de 2 prestataires par maternité. Il s'agissait d'une formation de formateurs qui ont par la suite assurée la formation du reste du personnel médical à Ouagadougou et Bobo Dioulasso.

\subsubsection{Organisation d'une journée d'information et d'orientation}

Une session d'information et d'orientation d'une journée sur le projet dans chaque site a été organisée pour 60 à 80 prestataires médicaux (médecins, sages femmes, infirmiers et assistantes sociales. L'objectif d'une telle réunion était d'obtenir l'adhésion du personnel des deux hôpitaux, de discuter de l'intégration de la nouvelle technologie de soins post abortum dans les activités régulières des deux maternités et d'informer les prestataires des autres centres de santé de la nouvelle approche de traitement des complications des avortements.

Il est important de souligner que les prestataires ont bénéficié d'évaluation formative à travers les visites de suivi des consultants de JHPIEGO.

\subsection{Intégration du counseling en PF dans la prise en charge des patientes}

Avant l'introduction du projet, il n'y avait presque pas de conseils en matière en $\mathrm{PF}$ dans le post-avortement a l'endroit des patientes sur le site; celles-ci étaient référées dans les $\mathrm{SMI} / \mathrm{PF}$ avec un risque de déperdition. La réorganisation des services a consisté à faire en sorte que les patientes admises pour avortement incomplet reçoivent dans le même établissement des prestations de services de qualité tant pour l'AMIU que pour le counseling en PF. Les services de gynécologie et obstétrique des deux hôpitaux ont mis en place chacun une unité d'AMIU au sein de la maternité. Deux sages-femmes ont été formées pour s'occuper de la préparation des patientes à l'AMIU et de la gestion du matériel au niveau de chaque site.

Circuit de la patiente selon le site

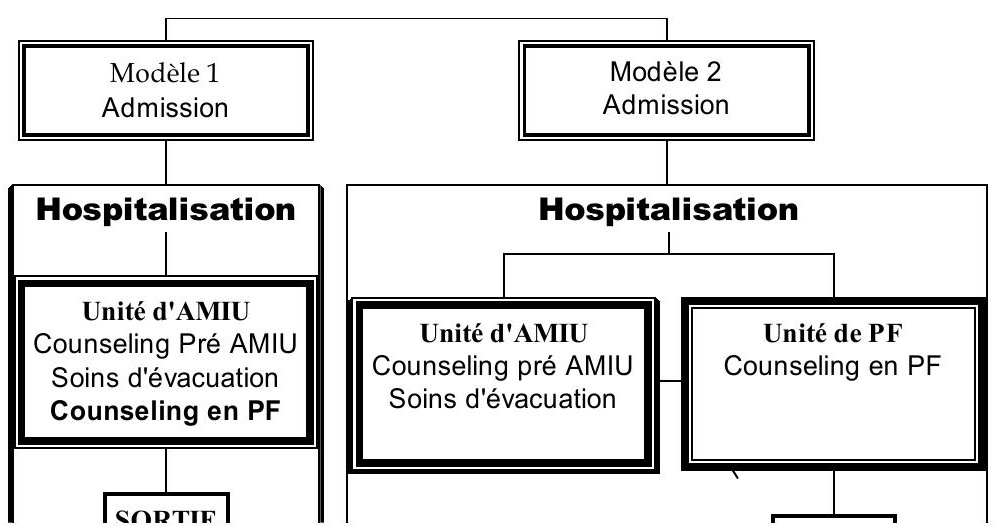


Cette réorganisation a été guidée par les suggestions des agents de santé des districts sanitaires de Ouagadougou et de Bobo Dioulasso1 lors des rencontres d'information et d'orientation. Il avait été suggéré que :

- La formation et l'utilisation de l'aspirateur manuel intra utérin s'adresse uniquement aux médecins.

- La prise en charge des patientes en counseling pré et post AMIU soit conduite par une sage-femme désignée et formée pour cette activité.

- Les prestations de PF soient conduites en plus du médecin, par une sage-femme.

- L'unité d'AMIU soit hors du bloc opératoire (lieu de la dilatationcuretage) et sa gestion soit sous le contrôle des supérieurs hiérarchiques et confiée à une ou deux sages-femmes.

- La gestion du matériel d'AMIU soit rigoureuse, au maximum le matériel sera disposé dans une armoire fermée à clé.

- L'unité d'AMIU soit placée en zone I d'asepsie tout comme le bloc opératoire.

C'est en prenant en compte ces recommandations des praticiens que le programme de gestion des SPA a été conduit avec une organisation légèrement différente selon le site.

\section{LES RESULTATS}

\subsection{Repartition de la Population EnQuetee}

Au total, au cours de la phase de collecte des données de base, 330 cas ont étés enquêtés dans les deux hôpitaux, dont 186 à Ouagadougou et 144 à BoboDioulasso. Quant à la post-intervention, 456 patientes ont pu être concernées, dont 223 à Ouagadougou et 233 à Bobo Dioulasso (tableau I).

En somme 119 prestataires ont participé à l'enquête comme indiqué dans le tableau I.

Tableau I : Répartition des patientes et les prestataires selon le site et la phase d'étude.

\begin{tabular}{l|c|c|c|c|c|c|c}
\hline & \multicolumn{3}{c|}{ OUAGADOUGOU } & \multicolumn{3}{c|}{ BOBO-DIOULASSO } & \multirow{2}{*}{ Totaux } \\
\cline { 2 - 9 } & Pré -test & Post-test & Total & Pré -test & Post-test & Total & \\
\hline \hline Clientes & 186 & 223 & 409 & 144 & 233 & 377 & $\mathbf{7 8 6}$ \\
Prestataires & 46 & 20 & 66 & 32 & 21 & 53 & $\mathbf{1 1 9}$ \\
\hline \hline
\end{tabular}

1. Le modèle de Ouagadougou est reconnu être plus approprié selon des résultats issus de plusieurs études, mais compte tenu des problèmes liés au personnel et aux travaux de construction en cours à la maternité de Bobo, le deuxième modèle a été adopté dans ce site. 


\subsection{Profil de la Population Enquetee}

\subsubsection{Caractéristiques socio-démographiques des patientes}

Notre échantillon d'étude de 786 patientes représentait 10,9 \% des patientes admises dans les deux maternités des hôpitaux au cours de la période de collecte.

Le profil socio-démographique des patientes était globalement similaire au cours des deux phases d'étude (avant et après intervention) et les différences observées n'étaient pas significatives.

\section{- Age des patientes}

Les patientes étaient relativement jeunes, $66,7 \%$ avaient moins de 30 ans. La tranche d'âge la plus touchée se situe entre 25 et 29 ans (27,0 \%). La moyenne d'âge était de 26 ans avec des extrêmes à 14 et 50 ans. La répartition des patientes par tranche d'âge est représentée par la figure 1 .

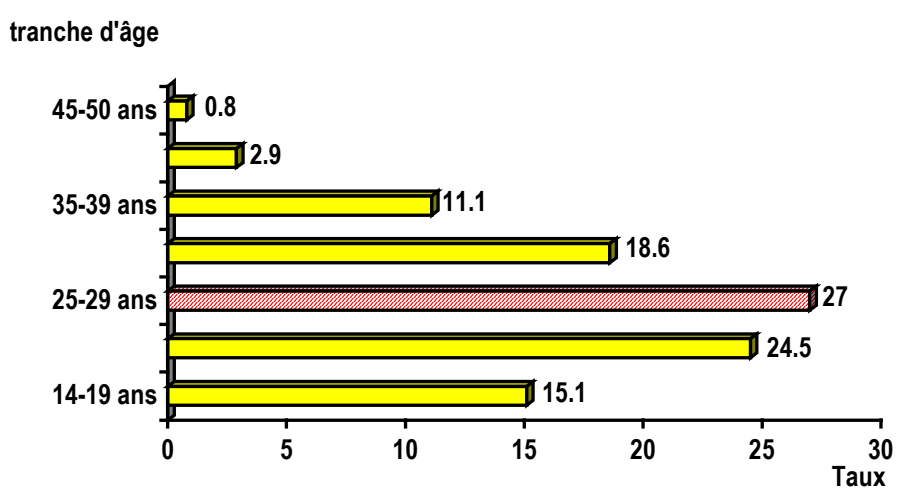

Figure 1: Repartition des patientes selon la tranche d'âge

Les adolescentes (1419 ans) représentent $15,1 \%$ de la population. Ce taux est sensiblement égal à celui relevé dans l'étude de la Bolivie $(15,9 \%)$ (JuanDiaz et Col) et sensiblement le double de celui rencontré dans l'étude d'Egypte (Nahla AbdelTawab) (8,5 \%) et du Sénégal (CEFOREP, PAC 1998) $(9,8 \%)$. Cette légère disparité des taux pourrait s'expliquer par l'âge de début de la vie sexuelle des femmes et aussi l'âge de mariage. Le risque d'exposition aux avortements existe à tous les âges de la vie active de femme, mais il reste, selon certaines données, plus marqué en seconde phase de cette vie sexuelle (20 à 34 ans). En effet ce groupe d'âge, représente $70,1 \%$ de notre échantillon d'étude, contre $14,8 \%$ des patientes d'âge supérieur à 35 ans.

- Niveau d'instruction

Le niveau d'instruction général était relativement bas (tableau II). Seulement, 39,1\% des patientes avaient un niveau d'instruction du secondaire et universitaire.

\section{- Statut matrimonial}

En ce qui concerne la situation matrimoniale, la grande majorité des patientes, soit $66,5 \%$ était marié. Les célibataires ne représentaient que $24,9 \%$; $41,2 \%$ des adolescentes sont mariées contre $66,4 \%$ des femmes d'âge compris entre 20 et 35 ans et $93,1 \%$ des femmes d'âge supérieur à 35 ans. 
- Profession

Pour $61,6 \%$ des femmes, les activités de ménage constituaient leur seule contribution à l'entretien de la famille. Les fonctionnaires représentent 14\%, les commerçantes $15 \%$ et le reste représentant les élèves et étudiantes.

\section{- Religion}

L'islam constituait la religion dominante avec un taux de 56,2 \% des patientes. Ce fort taux pourrait s'expliquer par la prédominance de cette religion au niveau du pays. Il existe une différence statistique $(p<0,001)$ par rapport aux phases d'étude quant à la religion.

\section{- Profil selon le site d'étude}

La distribution des patientes par tranche d'âge ou par groupes sociodémographiques ne subit pas de modification sensible selon que l'on les recrutait à Bobo Dioulasso (site 2) ou à Ouagadougou (site 1).

\section{$\underline{\text { Histoire reproductive des patientes }}$}

La toute première grossesse qui se termine par un avortement concerne $24,7 \%$ des patientes admises pour soins après avortement. Parmi les adolescentes, $75,6 \%$ sont à leur première grossesse contre $18,7 \%$ des femmes d'âge compris entre 20 et 34 ans.

Il est a remarquer que 40, $1 \%$ des adolescentes souhaitent avoir leur premier enfant après le mariage.

Par rapport à la parité, $28,8 \%$ des patientes n'ont jamais porté une grossesse à terme ; et parmi elles, 79,8 \% sont des adolescentes. Les paucipares (2 à 4 ) sont les plus concernées avec un taux de $34,8 \%$.

$\mathrm{Au}$ moment ou survenait la grossesse ayant conduit à cet épisode, seulement $6,7 \%$ des patientes utilisait une méthode contraceptive moderne ou naturelle (Tableau II). Les méthodes contraceptives modernes ayant connue un échec sont : la pilule (42,0\%), le condom (32,0\%) et les spermicides $(14,0 \%)$. 
Tableau II : Répartition des patientes selon leur histoire reproductive

\begin{tabular}{|c|c|c|c|}
\hline Caractéristiques & $\begin{array}{c}\text { Pré - Test } \\
N=330\end{array}$ & $\begin{array}{c}\text { Post }- \\
\text { Test } \\
\mathrm{n}=456 \\
\end{array}$ & $\begin{array}{c}\text { Total } \\
\mathrm{n}=786\end{array}$ \\
\hline $\begin{array}{l}\text { PARITE } \\
\text { Nullipare (0) } \\
\text { Primipare (1) } \\
\text { Paucipare (2-4) } \\
\text { Multipare (5- } 6) \\
\text { Grande Multipare }(\geq 7)\end{array}$ & $\begin{array}{r}29,1 \\
20,9 \\
35,2 \\
10,0 \\
4,8 \\
100\end{array}$ & $\begin{array}{r}28,5 \\
25,0 \\
34,6 \\
8,6 \\
3,3 \\
100 \\
\end{array}$ & $\begin{array}{r}28,8 \\
23,3 \\
34,8 \\
9,2 \\
3,9 \\
100\end{array}$ \\
\hline $\begin{array}{l}\text { UTILISATION PASSEE DE LA } \\
\text { PF } \\
\quad \text { Oui } \\
\end{array}$ & 52,5 & 52,8 & 52,7 \\
\hline $\begin{array}{l}\text { UTILISATION DE LA PF PDT } \\
\text { L'EPISODE } \\
\text { Méthode moderne } \\
\text { Méthode naturelle } \\
\text { Aucune méthode }\end{array}$ & $\begin{array}{r}6,7 \\
3,6 \\
89,7 \\
100\end{array}$ & $\begin{array}{r}6,1 \\
2,2 \\
91,7 \\
100\end{array}$ & $\begin{array}{r}6,7 \\
2,5 \\
90,8 \\
100\end{array}$ \\
\hline $\begin{array}{l}\text { PERIODE SOUHAITABLE POUR } \\
\text { LA NAISSANCE PROCHAINE } \\
<1 \text { an } \\
>1 \text { an } \\
\text { Après le mariage }\end{array}$ & $\begin{array}{r}\boldsymbol{N}=\mathbf{2 6 \boldsymbol { 6 }} \\
\\
29,3 \\
52,3 \\
18,4 \\
100\end{array}$ & $\begin{array}{r}n=385 \\
42,6 \\
40,3 \\
17,1 \\
100\end{array}$ & $\begin{array}{r}N=651 \\
\\
37,1 \\
45,2 \\
17,7 \\
100\end{array}$ \\
\hline
\end{tabular}

Parmi les patientes qui n'utilisaient pas un moyen anticonceptionnel moderne, seulement $39,6 \%$ avait un désir de grossesse.

\subsubsection{Connaissances des Patientes sur la période de la fécondité.}

La pratique de l'abstinence périodique ou méthode du calendrier menstruel comme moyen de régulation de la fécondité suppose pour la femme, une bonne connaissance de la période féconde. Cette connaissance peut être acquise au cours du cursus scolaire, au niveau de l'éducation sexuelle parentale ou au cours des prestations de $\mathrm{PF}$.

\section{- Période de fécondité}

Au cours de la première phase de l'étude (pré intervention), à la question "Quelle est la période du cycle menstruel ou la femme a plus de chance de tomber enceinte", seulement $43,9 \%$ des patientes la situent au milieu du cycle, et peuvent pratiquer l'abstinence périodique avec une chance théorique de succès. $\mathrm{Au}$ post test, le niveau de connaissance a connu une relative augmentation passant à $47,8 \%$. 
Il n'y a pas de différence statistiquement significative $(p=0,109)$ entre les deux phases d'étude quant au niveau de connaissance des patientes. Il apparaît donc qu'il y a une insuffisance d'informations au cours des prestations de PF, notamment dans le volet de la fécondité.

La méconnaissance de leur propre fécondité par les patientes est aussi

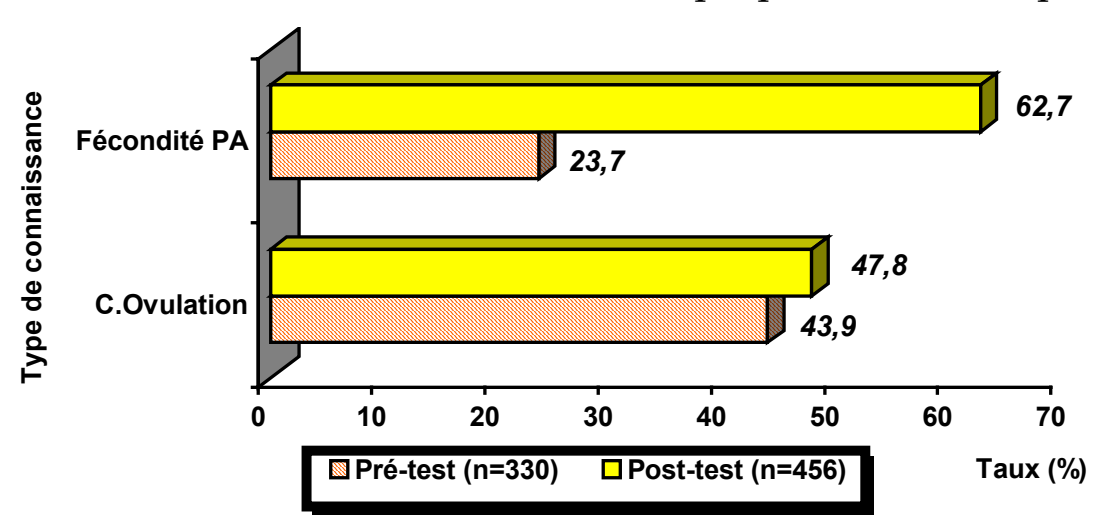
relevée dans les réponses données au cours du pré - test à la question évaluant la fécondité du post abortum (Figure 2). Seules $23,7 \quad \%$ des patientes situent cette fécondité avant le retour des règles au cours du pré test contre $62,7 \quad \% \quad$ au

Fig 2 : Niveau de connaissance des patientes

cours du post test. L'augmentation du taux de connaissance, du simple au double entre les deux phases d'étude indique non seulement une acquisition de connaissance des prestataires en matière de soins post abortum, mais aussi leur disponibilité à donner les informations sur la fécondité du post abortum aux patientes traitées dans les services.

Par rapport au niveau de connaissance sur la fécondité du post abortum, il existe une différence significative $(p<0,001)$ suivant les sites d'étude. Au niveau du site de Bobo Dioulasso, le niveau de connaissance des patientes est passé de $36,1 \%$ en première phase pour atteindre $69,5 \%$ en seconde phase ; Alors qu'à Ouagadougou, il est passé de $19,9 \%$ en première phase à seulement $55,6 \%$ en seconde phase.

\subsection{Données cliniques}

\subsubsection{Type d'avortement.}

Nous avons tenté de préciser le type d'avortement à l'aide des archives hospitalières ou par l'aveu de la patiente en se basant sur la définition de l'OMS, (WHO, 1987).

- Pour les avortements provoqués ; il a été reporté comme avortement provoqué suspect (IVG suspect), le cas de figure ou les archives relevaient un

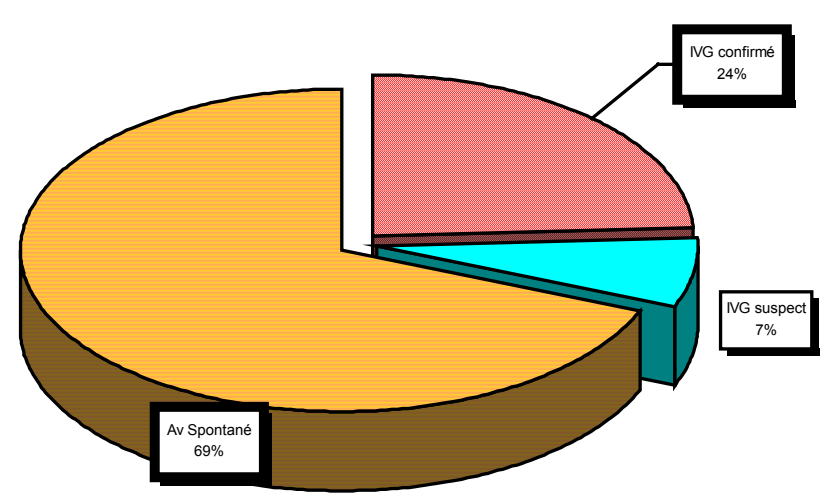
avortement provoqué ou une suspicion d'avortement provoqué avec un désaveu de la patiente. Les avortements provoqués avoués par la patiente ont été considérés comme tel (IVG confirmé). 
- Les autres cas ont été considérés comme avortement spontané.

Dans notre étude, les avortements spontanés constituaient la majeure partie soit 68,9 \% de notre échantillon (figure 3). La moyenne d'âge des patientes ayant subit un avortement spontané est de 27 ans contre 24 ans pour

les avortements provoqués. Selon l'âge, l'avortement provoqué $(54,4 \%)$ aussi bien que l'avortement spontané $(83,1 \%)$ se rencontraient le plus souvent chez une jeune femme mariée. Près de la moitié de ces patientes avaient moins de 25 ans (46,5\%). Les adolescentes (14-19 ans) représentent 18,5\% de cette population. Au niveau des adolescentes, l'avortement était provoqué dans $42,9 \%$ des cas.

Dans l'ensemble de notre échantillon, les avortements interviennent très précocement au cours de l'évolution de la grossesse, en moyenne à 9 semaines d'aménorrhée. $63,4 \%$ des patientes se recrutaient avant la 10 è semaine d'aménorrhée.

\subsubsection{Motif de consultation}

Le principal motif de consultation était les hémorragies génitales $(78,5 \%)$, ensuite viennent les douleurs pelviennes (13,7\%) et l'infection $(7,3 \%)$.

\subsection{Prise en charge des Patientes}

\subsubsection{Technique d'évacuation utérine}

Au cours de la phase de pré intervention, la dilatation curetage $(60,3 \%)$ et le curage digital (39,7 \%) étaient les deux techniques utilisées pour l'évacuation des débris ovulaires dans les avortements incomplets (tableau III).

En seconde phase d'étude l'AMIU a constitué majoritairement (97,1\%) la technique utilisée au détriment de la dilatation curetage $(1,8 \%)$ et du curage digital (1,1 \%). Mais, l'approche des prestataires dans la prise en charge des patientes $\mathrm{du}$ post abortum semble ne pas exclure totalement une des trois techniques.

Dans cette approche, les soins d'évacuation des débris ovulaires par dilatation curetage (5 cas) ou par curage digital (9 cas) ont été conduits pour stabiliser l'état clinique de ces patientes surtout en dehors des heures d'ouverture des unités d'AMIU.

\section{TABLEAU III: Répartition des patientes selon la technique}

\begin{tabular}{l|c|c}
\hline \hline TECHNIQUE & PRE- & POST-TEST \\
D'EVACUATION & TEST & $\boldsymbol{N}=\mathbf{4 5 6}$ \\
UTERINE *** & $\mathbf{n}=\mathbf{3 3 0}$ & \\
Curage digital & 39,7 & 1,8 \\
Dilatation - Curetage & 60,3 & 1,1 \\
AMIU & 0,0 & 97,1 \\
\hline \hline
\end{tabular}




\subsubsection{Procédure de Contrôle de la Douleur}

$23,0 \%$ de cas de dilatation curetage se sont faits sous anesthésie générale au bloc opératoire, mobilisant une équipe de 3 à 4 agents de santé.

$12,2 \%$ des cas de curage digital faite en salle d'admission ont pu bénéficiés d'un antalgique après la manœuvre. Dans le reste des cas, la procédure a été conduite sans antalgique avant, pendant et après la manœuvre.

Quant à l'aspiration manuelle intra utérine elle a été conduite dans 98,0 \% des cas grâce à une préparation psychologique des patientes encore appelée «anesthésie verbale». Malgré cette préparation, 27,1\% des cas ont bénéficié d'une anesthésie loco-régionale avant la manœuvre et 6,6 \% d'un antalgique après la manœuvre.

\subsubsection{Perception de la douleur par les patientes}

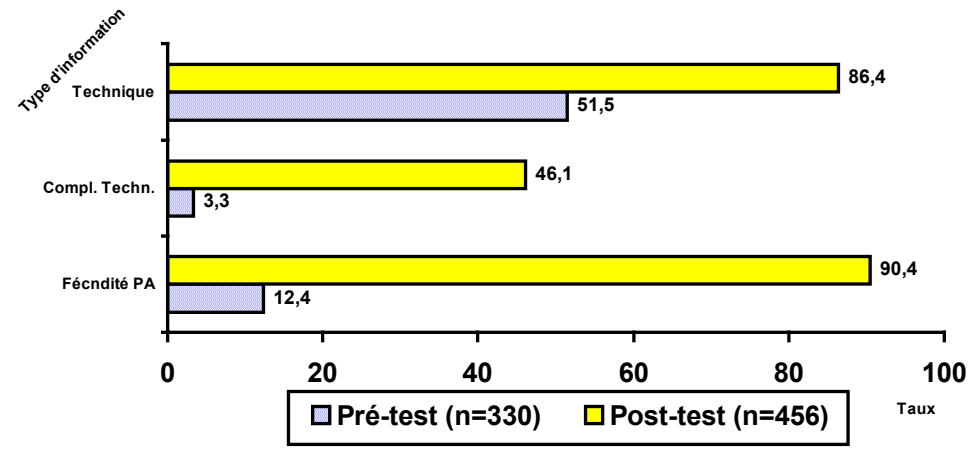

Figure 4 : Informations données aux patientes

Nous avons estimé la perception de la douleur suivant 4 degrés.

- La perception de douleur extrême correspondait à un seuil selon lequel la patiente perçoit une douleur au point d'exiger l'arrêt de la procédure ou de

l'administration d'un antalgique.

- La perception de picotement correspondait à un seuil de douleur minime.

- La douleur était modérée, lorsqu'elle était tolérable, à la limite des deux précédentes.

- Aucune douleur correspondait à l'absence d'une perception de douleur, de gène sinon d'une sensation évoquant un examen gynécologique courant.

Dans le cas de dilatation curetage, les patientes n'ont perçu aucune douleur. Par contre, au cours du curage digital la douleur était extrême pour 71,9 $\%$ des patientes.

La majorité des patientes (98,0 \%) ayant bénéficié de la technique d'AMIU ont reçu une préparation psychologique avant et pendant la manœuvre afin de supporter la douleur. Pour $69,0 \%$ des patientes la douleur était tolérable et ne nécessitait pas l'administration d'antalgique après la manœuvre.

L'analyse des données sur la perception et le contrôle de la douleur permet de dire tout comme Kizza au Kenya, qu'un soutien verbale bien conduit permettrait aux patientes de mieux se mettre en confiance et de supporter la douleur lors de la procédure d'AMIU. 


\subsubsection{Informations reçues par les Patientes.}

Un des éléments clés de la qualité des soins de santé repose sur la communication entre prestataire et patiente. Il est donc essentiel qu'en plus des soins curatifs, les agents de santé donnent des conseils aux patientes par rapport à leur état morbide, répondent aux questions et lèvent leurs inquiétudes.

Dans notre étude au cours de la phase de pré-intervention, les informations sur la fécondité du post abortum (fécondité PA), les complications liées à technique d'évacuation utérine ont été rarement données aux patientes.

\section{- Technique d'évacuation}

Trois patientes sur quatre ont été informées de la technique d'évacuation du contenu utérin que l'on a adopté pour leurs soins. Cette information a surtout été donnée au cours de la seconde phase

\section{- Survenue de complications}

$\mathrm{Au}$ cours de leur séjour hospitalier, la plupart des patientes de la première phase d'étude ont été immédiatement libérées après la procédure d'évacuation utérine sans conseils particuliers sur les problèmes sanitaires du post abortum. En effet, l'information sur la possibilité de survenue de complications liées à la technique utilisée n'a été donnée qu'à $3,3 \%$ des patientes en première phase contre $46,1 \%$ en seconde phase d'étude.

\section{- Fécondité du post abortum}

Quant à la fécondité du post abortum, au cours de la première phase d'étude, seulement $12,7 \%$ des patientes contre $90,4 \%$ en seconde phase ont été informées sur la fécondité du post abortum immédiat.

La différence très marquée entre les deux phases d'étude quant aux informations relatives aux complications des soins d'évacuation utérine et à la fécondité du post abortum se justifie par l'acquisition des connaissances de la part des prestataires après la formation en soins post abortum

Selon le site d'intervention et cela en seconde phase d'étude il existe une différence significative entre le CHN-SS et le CHN-YO quant à la qualité des prestations de soins post abortum.

Les patientes ont bénéficié des informations sur la fécondité du post abortum (94,8 \% - p<0,001) au CHN-SS qu'au CHN-YO (respectivement 80,7 \% et 85,7 \%). Par contre, le site du CHN-YO a été plus disposé à informer les patientes sur les complications $(57,0 \%-\mathrm{p}<0,001)$ pouvant survenir après les soins d'évacuation que celui de Bobo-Dioulasso (35,6 \%). Quant à l'information sur la technique de soins il n'existe pas de différence significative entre les deux sites d'étude $(\mathrm{p}<0,002)$.

\subsection{Durée d'hospitalisation}

Le temps d'attente après l'admission et le premier examen est relativement court pour la grande majorité des patientes. La prise en charge 
diagnostic et les premiers soins ont été effectifs dans la première heure d'admission pour $82,1 \%$ des patientes.

La durée moyenne d'hospitalisation pour l'ensemble de notre échantillon d'étude est de 1,124 jour soit 27 heures de séjour moyen hospitalier, avec des extrêmes de moins d'une journée à 10 jours. 77,0 \% des patientes ont bénéficié de soins post abortum dans les 24 heures après leur admission dans le service d'urgence.

Cette durée moyenne variait de manière significative suivant la technique utilisée (figure 4) pour l'évacuation des débris ovulaires. Une moyenne de 19 heures $(0,81$ jour) est relevée au cours de l'utilisation de l'AMIU contre 39 heures (1,65 jour) pour la technique de dilatation-curetage et 31 heures $(11,31$ jour) dans les cas de curage digital.

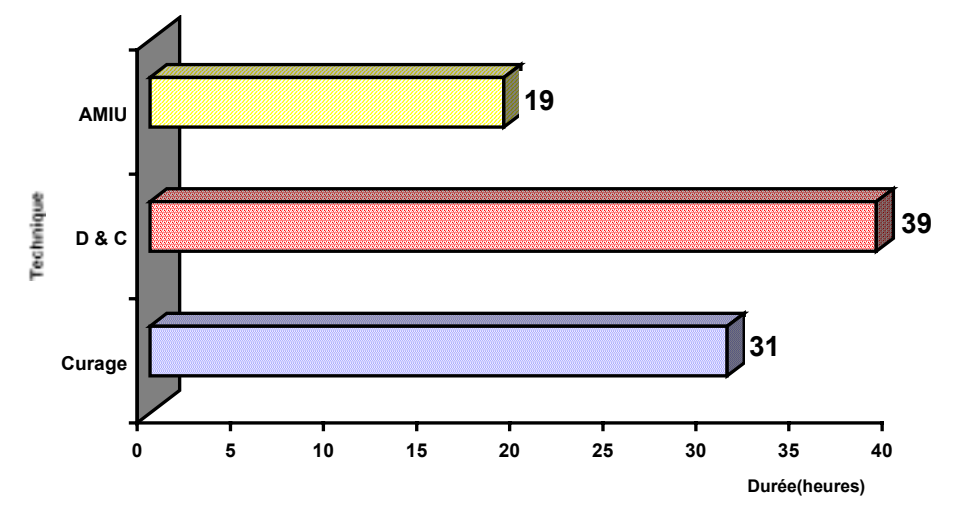

L'utilisation de l'AMIU comme technique de soins post abortum offre une plus courte durée d'hospitalisation aux patientes, d'où une réduction des frais de prise en charge pour les hôpitaux.

Figure 5 : Durée moyenne d'hospitalisation selon la technique

Comme le montre la figure 5, on constate que le site du CHN-YO, où les services de prestation de $\mathrm{PF}$ sont intégrés aux prestations d'AMIU, 62,2 $\%$ des patientes ont pu être libéré le jour de leur admission contre seulement $39,8 \%$ sur le site du CHN-SS.

La durée moyenne est de $1,12 \mathrm{j}$ pour le CHN-SS contre $0,51 \mathrm{j}$

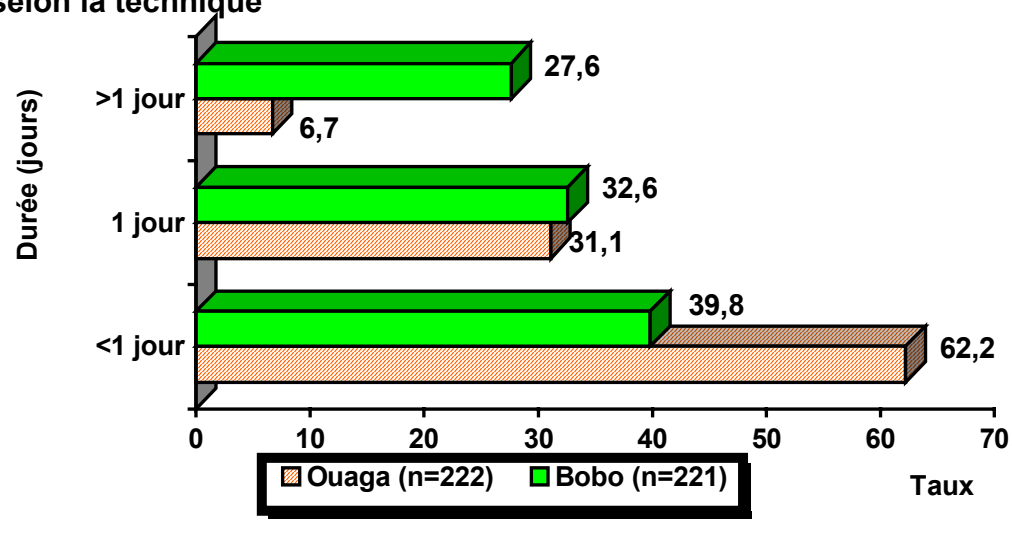

Figure 6 : Durée d'Hospitalisation en cas d'AMIU selon le site pour le CHN -YO.

Il apparaît donc, que la séparation des prestations de PF aux prestations d'AMIU garantirait plus un counseling en PF mais rallonge la durée de séjour hospitalier des patientes.

\subsection{Satisfaction des patientes}

La majorité des patientes $(91,4 \%$ ) admises pour soins après avortement est satisfaite des prestations de services au cours de leur séjour hospitalier. La qualité des prestations de services a été plus appréciée positivement (93,6\%) au cours de la seconde phase d'étude qu'en phase de pré intervention. La différence était significative $(\mathrm{p}=0,0003)$.

Néanmoins, l'élévation du niveau de connaissance et l'amélioration de la santé des patientes avec une réduction de la durée moyenne de séjour sont la 
résultante d'une amélioration des services de santé. L'impact réel est la satisfaction des bénéficiaires du système ; c'est à dire les patientes.

TABLEAU IV : Niveau de satisfaction des patientes.

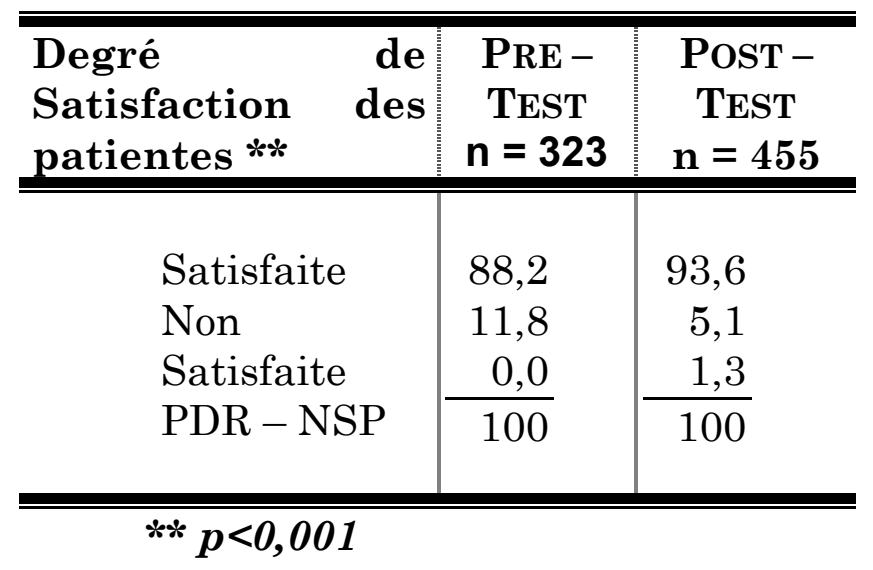

$$
\begin{aligned}
& \text { L’appréciation de } \\
& \text { la qualité d'une } \\
& \text { prestation par le } \\
& \text { bénéficiaire lui- } \\
& \text { même est difficile } \\
& \text { dans le contexte } \\
& \text { culturel africain. }
\end{aligned}
$$

Pour éviter de vexer son interlocuteur, la patiente répondrait volontiers qu'elle est satisfaite ce qui constituait un biais de courtoisie. Dans ce contexte le faible taux général de 6,1 \% d'insatisfaction refléterait très peu l'avis des patientes. Cette approche se justifie par les suggestions faites par les patientes pour l'amélioration de la qualité des soins. En effet, 613 patientes soit un taux de 77,9 \% suggèrent des améliorations dans la qualité des prestations de soins. Ces amendements intéressent tant bien l'accueil (40,5\%) que la disponibilité du personnel de santé $(23,7 \%)$ ou la compréhension des patientes par le prestataire.

\subsection{Planification familiale chez les patientes}

\subsubsection{Connaissance de la Planification Familiale}

La série de questions évaluant le niveau de connaissance général des patientes sur la PF révèlent un niveau relativement élevé.

La majorité des patientes (95,3\%) connaît l'existence des moyens et méthodes qu'un couple pourrait utiliser pour éviter ou retarder une grossesse.

Spontanément, $64,3 \%$ des patientes citent trois méthodes la pilule et le dispositif intra utérin viennent en première position (57,9 \%) avec le plus souvent le condom ou les injectables.

$20,8 \%$ des patientes ne peuvent citer que deux méthodes avec une connaissance plus marquée pour la pilule $(93,0 \%)$ et le condom $(76,4 \%)$ ou la pilule et les injectables $(10,8 \%)$.

Seulement 14,9 \% des patientes ne connaissent qu'une seule méthode contraceptive connue.

Une faible proportion des patientes $(6,5 \%)$ connaissent le Norplant comme méthode contraceptive moderne.

Les méthodes contraceptives irréversibles sont peu connues.

Il y a une différence statistiquement $(\mathrm{p}<0,001)$ significative entre le niveau de connaissance des méthodes contraceptives modernes en seconde phase (98,5\%) de l'étude qu'en première phase (91,5\%). L'introduction du counseling en PF dans les soins post abortum justifierait cette différence entre les deux phases d'étude. Mais le bas niveau de connaissance des méthodes irréversibles de contraception et du Norplant serait probablement la résultante d'une 
présentation limitée des méthodes modernes lors du counseling aux clientes de la $\mathrm{PF}$ en général.

- Selon le statut matrimonial

Par rapport au statut marital, les patientes mariées ont une meilleure connaissance des méthodes contraceptives modernes (66,2\%). Ce sont elles qui plus volontiers citent les condoms et le Norplant comme moyens contraceptifs modernes.

\section{- En fonction de l'âge}

On enregistre une grande disparité du niveau de connaissance des méthodes contraceptives modernes selon l'âge des patientes. Les adolescentes (18,5\%) et les femmes d'âge supérieur à 40 ans (20,6 \%) sont celles qui semblent connaitre le moins la contraception moderne. Il semble exister une information discriminatoire selon l'âge pour la présentation de la diversité des méthodes contraceptives modernes présentées lors des prestations de PF.

\section{Source d'information}

La plupart des patientes ont eu la première information sur les méthodes contraceptives par le biais d'une amie ou un parent (41,3\%) ou auprès des structures sanitaires $(30,5 \%)$. Seulement, $2,6 \%$ des patientes ont reçu l'information sur la planification familiale par l'intermédiaire de leur partenaire ou mari.

\subsubsection{Intention d'utiliser la Planification Familiale}

Pratique contraceptive passée : Dans notre échantillon d'étude, la moitié des patientes $(52,7 \%)$ a déjà utilisé une méthode de contraception moderne à un moment quelconque de leur vie. La méthode moderne anticonceptionnelle la plus utilisée était la pilule (68,0\%), suivi du condom (16,8\%) et du dispositif intra utérin $(6,7 \%)$.

Dans le passé contraceptif des patientes le choix de la méthode contraceptive moderne semble avoir été guidé par leur statut marital. En effet, les patientes en union constituent le groupe qui a le plus utilisé une méthode contraceptive moderne $(67,0 \%)$, suivi des patientes célibataires $(24,0 \%)$. La méthode moderne la plus utilisée par les patientes mariées est la pilule $(60,3 \%)$ suivi du DIU (6,1\%).

En ce qui concerne les patientes célibataires, elles ont utilisé dans le passé en proportion sensiblement égale la pilule (37,5\%) et le condom (36,5\%).

Utilisation future: $\mathrm{Au}$ cours de leur séjour hospitalier, 67,3 \% des patientes ont pu bénéficier de conseils en planification familiale. Comme le montre le tableau $\mathrm{V}$, les prestations de planification familiale ont été dispensées en seconde phase d'étude pour $94,3 \%$ des femmes contre seulement $30,3 \%$ en première phase. Pour les patientes ayant bénéficiés de prestations de PF, 81,8 \% voudraient utiliser dans le future une méthode contraceptive moderne en post intervention contre seulement $63,9 \%$ en pré - intervention. 


\section{Tableau V : Prestations de la PF / Counseling à l'intention des patientes}

\begin{tabular}{lr|l|l}
\hline \hline $\begin{array}{l}\text { COUNSELING } \\
\text { INTENTION D’UTILISER }\end{array}$ & ET & PRE - TEST & $\begin{array}{c}\text { POST - } \\
\text { TEST }\end{array}$ \\
\hline \hline $\begin{array}{l}\text { COUNSELING EN PF DONNEE ** } \\
n=786\end{array}$ & 30,0 & 94,3 \\
\hline $\begin{array}{l}\text { UTILISATION FUTURE DE LA } \\
\text { PF** } \\
\mathrm{n}=526\end{array}$ & 63,9 & 81,8 \\
$\begin{array}{l}\text { METHODE CONTRACEPTIVE } \\
\text { RECUE*** } \\
\mathrm{n}=524\end{array}$ & 56,7 & 83,1 \\
\hline \hline
\end{tabular}

Soixante sept pour cent $(67,3 \%)$ des patientes ont reçu un counseling en $\mathrm{PF}$ dont $30,0 \%$ des patientes en période de pré - intervention et $94,3 \%$ en post intervention. De ces clientes, 3 sur $4(78,5 \%)$ après le Counseling en planification familiale ont accepté de commencer l'utilisation d'une méthode contraceptive moderne. L'utilisation future et l'acceptation actuelle d'une méthode contraceptive moderne sont proportionnelle à la participation d'un counseling en $\mathrm{PF}$ comme le montre le tableau $\mathrm{V}$ ci-dessus.

Le passé contraceptif n'a pas d'influence sur l'utilisation future de la PF, mais le counseling en PF joue un rôle majeur dans le recrutement de nouvelles clientes. Cette remarque se vérifie dans l'analyse comparative des prestations en $\mathrm{PF}$ et les intentions futures d'utiliser la PF au niveau des patientes. En effet, 96,9 \% des patientes ayant bénéficié d'un counseling en $\mathrm{PF}$ ont l'intention d'utiliser une méthode moderne de PF contre seulement $43,9 \%$ des patientes n'ayant pas eu le bénéfice d'un counseling.

\section{- L'âge}

Dans notre étude, nous constatons que les besoins en matière de PF ne sont pas seulement l'apanage des patientes d'un âge mur, mais intéresse aussi les adolescentes (14-19 ans). En effet, 77,4 \% des adolescentes de notre échantillon, a l'intention d'utiliser une méthode contraceptive moderne, contre $80,1 \%$ des patientes de la tranche 20 à 34 ans et $71,8 \%$ des patientes d'âge supérieur ou égal à 35 ans. 


\section{Parité et nombre d enfants vivants}

Contrairement à ce qui aurait été logique, le nombre élevé d'enfants par patiente ne joue pas un rôle majeur dans leur comportement en matière de contraception. On constate (figure 7) que l'intention d'utiliser une méthode contraceptive moderne est sensiblement le même quelque soit le nombre d'enfants vivants par patiente.

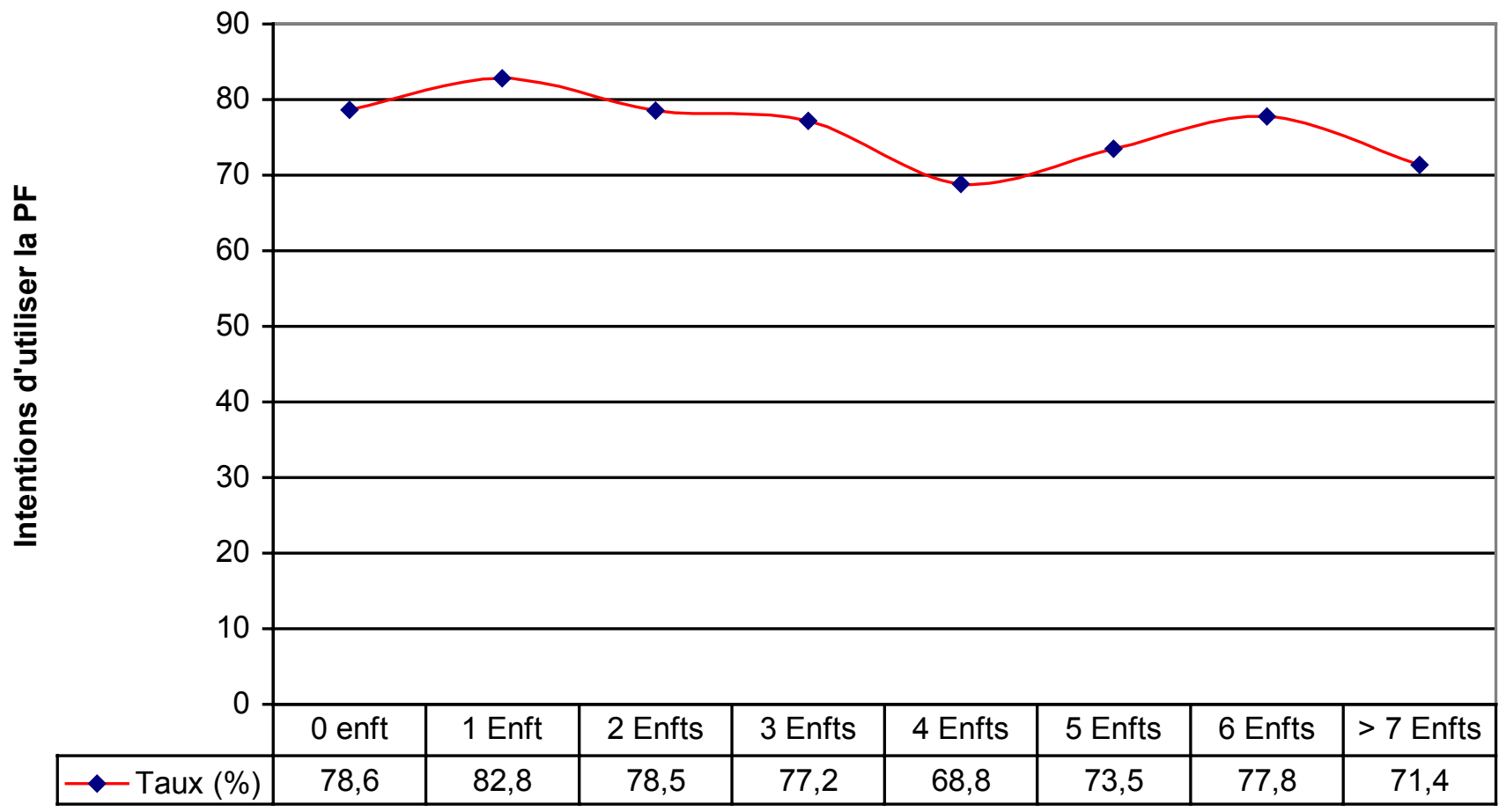

Nombre d'enfants par patiente

Figure 7 : Tendences des intentions d'utiliser la PF par rapport au nombre d'enfants vivants par patiente

Ce fait pourrait s'expliquer par la jeunesse de notre échantillon, qui a de plus en plus des idées moins natalistes, par rapport à la conception pro - nataliste de la femme africaine. De plus, la taille de la famille est très souvent décidée par le partenaire en Afrique.

\subsubsection{Acceptation de la Planification Familiale}

Le désir de ne pas utiliser dans l'avenir une méthode moderne de contraception est justifié par diverses raisons par les patientes :

- La convenance personnelle est la première raison évoquée par 113 patientes $(49,0 \%)$.

- Le désir d'avoir un enfant est la seconde raison évoquée (15,0 \%) quel que soit les caractéristiques socio-démographiques analysées. 
- La peur des effets secondaires (11,0 \%), l'opposition du mari/conjoint (6,0 \%) ou l'irrégularité des rapports sexuels $(3,0 \%)$ sont autant de raisons motivant la non utilisation très prochaine de moyens anticonceptionnels modernes.

La quasi - totalité des patientes (95,0 \%) ayant bénéficié de conseils en planification familiale trouve le lieu, le moment et la qualité des prestations acceptables.

Bien que 89,4\% des patientes approuve la planification familiale, seulement 410 patientes ont accepté recevoir une méthode contraceptive moderne, soit un taux d'acceptabilité de 78,2\% (n=526).

Le niveau d'acceptabilité d'une méthode contraceptive moderne après le counseling en $\mathrm{PF}$ a été plus élevé en phase de post intervention (83,1\%) qu'en pré intervention (56,7\%).

Le site de Ouagadougou recrutait plus de patientes utilisatrices d'une méthode contraceptive moderne (90,4 \%) que celui de Bobo-Dioulasso (70,2\%).

Par rapport à la tranche d'âge, les adolescentes ont autant accepter une méthode contraceptive moderne (76,2 \%) que les femmes d'âge mur ; 80,2 \% pour la tranche d'âge de 20-30 ans et 70,4\% pour les femmes d'âge supérieur ou égal à 35 ans.

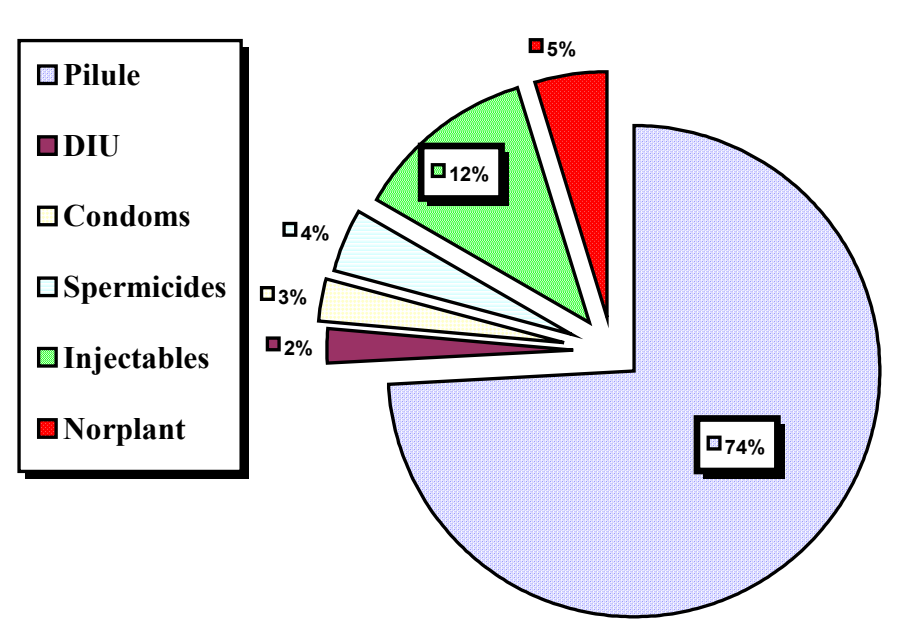

Figure 8 : Type de méthode Contraceptive récu par les patientes $(n=410)$

Les trois quarts des patientes ont reçu la pilule (figure 8) comme méthode contraceptive moderne. Parmi les autres méthodes, la demande en injectable intéressait $12,0 \%$ des patientes et le Norplant 4,6\%.

Par contre selon le site, les patientes du CHN-YO ont plus porté leur choix vers la pilule $(85,7 \%)$ que celles du site du CHN-SS (64,3\%), alors que, les patientes du CHN-SS ont eu un choix plus varié quant à la méthode contraceptive, notamment les injectables (19,0\% contre $3,7 \%)$, le Norplant $(7,2 \%$ contre $1,1 \%)$. Cette possibilité de choix varié au niveau du site de CHN-SS doit être due à une grande variété des méthodes contraceptives présentées aux patientes.

\subsubsection{Qualité des prestations}

La formation des prestataires en technologie contraceptive a eu un impact réel sur la qualité du counseling en PF. Cette amélioration est plus relevée sur le site du CHN-SS que celui du CHN-YO (tableau VI). Le mode d'utilisation des méthodes contraceptives offertes aux patientes à été plus expliqué en seconde phase d'étude qu'en première. Dans l'ensemble, les patientes sont très rarement informées sur la conduite à tenir lors du changement de méthode contraceptive. 
TABLEAU VI: Qualité des prestations en planification familiale en post test selon le site

\begin{tabular}{|c|c|c|}
\hline $\begin{array}{lll}\text { Counseling } & \text { et } & \text { intention } \\
\text { d'utiliser la PF } & & \\
\end{array}$ & $\begin{array}{l}\text { OUAGA } \\
\mathrm{n}=202\end{array}$ & $\begin{array}{l}\text { Вово } \\
\mathrm{n}=227\end{array}$ \\
\hline FACILITE DE COMPREHENSION & 92,6 & 98,7 \\
\hline $\begin{array}{l}\text { Possibilite DE POSER DES } \\
\text { Questions }\end{array}$ & 96,5 & 100 \\
\hline $\begin{array}{l}\text { EXPLICATION SUR LE } \\
\text { FONCTIONNEMENT }\end{array}$ & $\begin{array}{l}88,3 \\
98,9\end{array}$ & $\begin{array}{r}100 \\
99,4\end{array}$ \\
\hline MODE D'UTILISATION & 35,6 & 66,9 \\
\hline 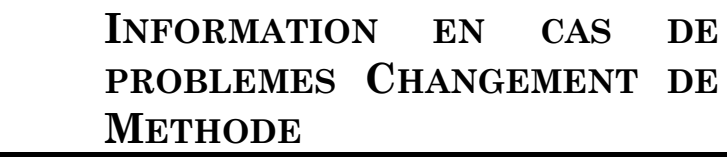 & 30,3 & 15,7 \\
\hline
\end{tabular}

\subsubsection{Implication du conjoint à la planification familiale.}

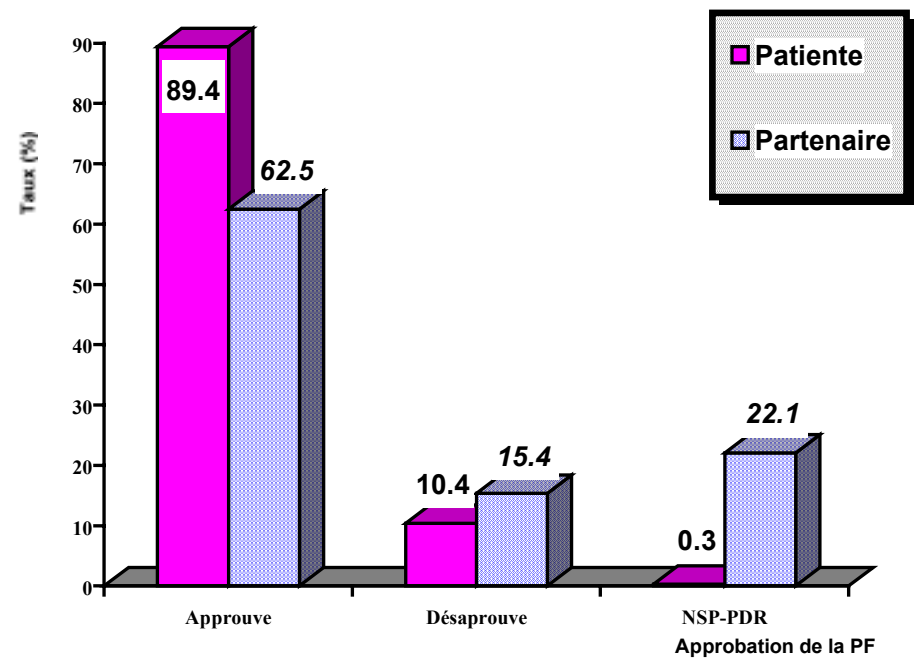

Figure 9 : Approbation de la femme et approbation de I'homme (selon la femme)

La planification familiale est approuvée par une grande partie des patientes $(89,4 \%)$. Un des points essentiels au succès d'un programme de planification est l'implication du conjoint aux prestations et au choix de la méthode contraceptive. Il est donc important, que les couples discutent entre eux et proposent la méthode contraceptive qui leur convient le mieux lors des

prestations.

Pour apprécier cet aspect nous avions posé des questions indirectes à la patiente afin analyser l'état d'esprit du couple et évaluer l'implication des hommes aux prestations de planification familiale. Ainsi, à la question «Pensezvous que votre mari ou partenaire approuve la planification familiale», 3 femmes sur 5 estiment qu'il l'approuve. 
La communication entre partenaires sur la planification familiale a été très régulière et s'est établi dans $69,5 \%$ des cas. On constate que l'approbation de la PF par le couple est plus élevée lorsque le sujet a été déjà abordé dans le foyer. Cette tendance pourrait

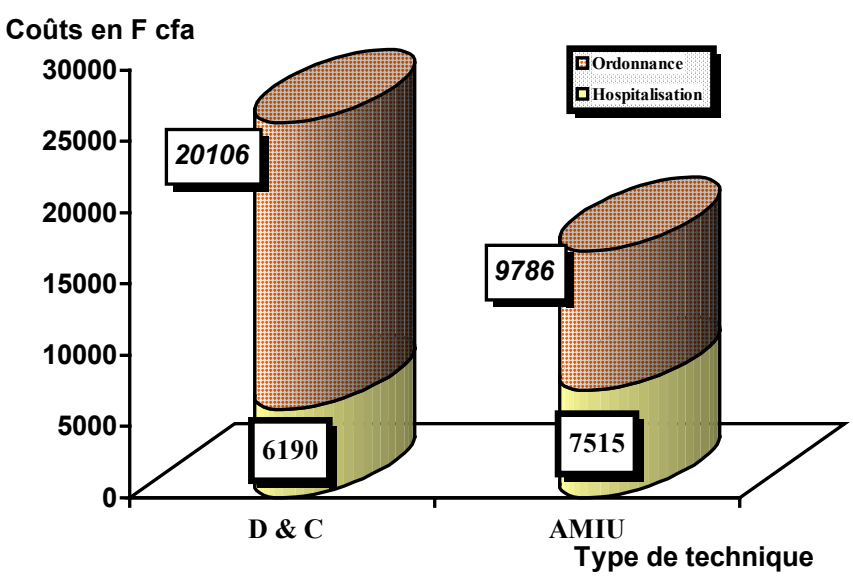

Figure 10 : Coûts moyens des dépenses supportées par la patiente selon la technique expliquer le taux élevé de patientes $(83,6 \%)$ qui désirent que leur partenaire participe aux prestations de planification familiale.

L'approbation de la planification familiale par le conjoint/partenaire varie suivant certaines variables socio-démographiques.

- Selon le statut marital, les patientes mariées $(64,8$ $\%)$ et celles vivant en concubinage $(69,5 \%)$ sont celles qui pensent le plus que leur époux/partenaire approuve la planification familiale par rapport au célibataires (54,6\%).

- Selon le niveau d'instruction, le conjoint approuve la PF chez 77,3 \% des patientes du niveau secondaire et $85,1 \%$ des patientes du niveau supérieur. Chez les patientes sans instruction, seulement $50,7 \%$ des conjoints qui approuvent la PF. Ceci s'explique par l'influence qu'à le niveau d'instruction du couple et surtout de la femme dans l'établissement de la communication avec son époux.

- Selon la religion: Les partenaires des femmes catholiques $(70,0 \%)$ et protestantes $(71,4 \%)$ approuvent la PF contre $58,4 \%$ pour les musulmanes.

" Nous sommes mis à l'écart et n’intervenons que pour honorer les ordonnances; même si c'est une histoire de femme, il faut au moins nous informer sur certains aspects des soins". C'est en ces termes que s'exprimait un époux qui assistait son épouse hospitalisée pour soins post abortum. Cette demande implicite d'informations sur toutes les étapes de soins, notamment au cours des prestations de planification familiale pourrait être le souhait de tous les partenaires comme le soulignent $82,2 \%$ des patientes interviewées.

Nous constatons que le taux d'approbation de la planification familiale est plus élevé lorsque les couples ont déjà discuté du sujet $(80,1 \%)$ que lorsque le sujet n'a jamais été abordé (19,0\%). Mais quelle est la part réelle de l'homme dans le choix et l'utilisation d'une méthode contraceptive moderne par le couple?

$\mathrm{Au}$ Burkina, surtout au sein des communautés rurales, les hommes ne veulent pas discuter d'un sujet tel que la planification familiale sur lequel ils sont moins informés que leur épouse (GUE et Coll.). Il apparait donc que les hommes bien informés pourront discuter avec leur femme et aider celles-ci à évaluer les besoins et à choisir une méthode de planification familiale.

De nombreuses études relèvent la part importante des hommes dans le choix, le suivi et la continuation d'une méthode contraceptive moderne. De plus, l'analyse 
de la plupart des données de l'EDS (Enquêtes démographiques de santé) en Afrique indique que c'est le mari qui est le véritable décideur des questions de fécondité.

Il apparaît important de faire participer le conjoint/partenaire aux prestations en PF pour non seulement augmenter le taux d'acceptabilité de la PF, mais surtout pour élever son niveau de connaissance en PF ce qui lui permettra d'entamer à l'aise une discussion à ce sujet avec son épouse/ partenaire. Cette approche pourrait aussi avoir un effet positif sur l'éducation sexuelle en milieu familial.

\subsection{LES PRESTATAIRES DES SERVICES}

\subsubsection{Caractéristiques socio-démographiques et professionnelles}

Les résultats révèlent que la plupart du personnel impliqué dans les soins après avortement sont de sexe féminin ; en effet, les femmes représentent respectivement $66,7 \%$ et $70,7 \%$ en pré-test et en post test. Aussi dans les deux phases de l'étude, les sages-femmes et demeurent les plus nombreuses parmi les prestataires interviewés

L'âge moyen des prestataires n'a pas varié entre les deux phases de l'étude, le pré-test et le post test.

Tant en pré-test qu'en post test, la proportion des prestataires mariés reste la plus importante comme l'indique le tableau VII.
Tableau VII : Caractéristiques sociodémographiques du personnel

\begin{tabular}{|c|c|c|}
\hline & $\begin{array}{l}\text { Pré-test } \\
(n=78)\end{array}$ & $\begin{array}{l}\text { Post-test } \\
(n=41)\end{array}$ \\
\hline \multicolumn{3}{|l|}{ Sexe } \\
\hline Masculin & 33,3 & 29,3 \\
\hline Féminin & 66,7 & 70,7 \\
\hline \multicolumn{3}{|l|}{ Age } \\
\hline Age moyen & 37,49 & 37,78 \\
\hline \multicolumn{3}{|c|}{ Situation matrimoniale } \\
\hline Marié & 79,5 & 68,3 \\
\hline Célibataire & 11,5 & 24,4 \\
\hline Autre & 9,0 & 7,3 \\
\hline
\end{tabular}

\subsubsection{Formation en soins post abortum}

En première phase, seulement 9\% des prestataires interviewés avaient déclaré avoir reçu une formation en AMIU et 7\% avaient reçu avaient eu une expérience directe avec le MVA, soit en ayant aidé un autre prestataire à en pratiquer ou en l'ayant pratiqué soi-même.
Tableau V III : Formation en AMIU

\begin{tabular}{|l|l|l|}
\hline & $\begin{array}{l}\text { Pré-test } \\
(n=78)\end{array}$ & $\begin{array}{l}\text { Post- } \\
\text { test } \\
(n=41)\end{array}$ \\
\hline $\begin{array}{l}\text { Formation en } \\
\text { AMIU }\end{array}$ & 9 & 49 \\
\hline \multicolumn{2}{|l|}{} \\
\hline $\begin{array}{l}\text { Expérience } \\
\text { pratique en AMIU }\end{array}$ & 7 & 63 \\
\hline
\end{tabular}


L'introduction de l'AMIU dans la seconde phase a contribué à améliorer énormément la situation. En effet, $49 \%$ des prestataires enquêtés lors de la phase 2 ont pu recevoir une formation sur l'AMIU et $63 \%$ d'enter eux en ont eu une pratique directe.

\subsubsection{Formation en planification familiale après avortement}

L'introduction des soins après avortement a contribué à la disponibilisation de l'information et des services de planification au sein de la maternité des deux hôpitaux concernés par l'étude. Pendant le pré-test, $45 \%$ des prestataires étaient formés en $\mathrm{PF}$ tandis que ce chiffre est passé à $71 \%$ en deuxième phase. C'est certainement l'effet de la mise à jour en technologie contraceptive.
Tableau IX : Informations et prestations de services PF

\begin{tabular}{|l|l|l|}
\hline & $\begin{array}{l}\text { Pré- } \\
\text { test } \\
(n=78)\end{array}$ & $\begin{array}{l}\text { Post- } \\
\text { test } \\
(n=41)\end{array}$ \\
\hline Formation en PF & 45 & 71 \\
\hline $\begin{array}{l}\text { Formation en PF } \\
\text { post avortement }\end{array}$ & 27 & 51 \\
\hline
\end{tabular}

Egalement, si $27 \%$ des prestataires interviewés en première phase disaient avoir reçu une formation en PF post avortement, en post test ils sont presque deux fois plus nombreux à avoir reçu cette formation.

Ces constations contribuent à l'augmentation de la qualité des soins post abortum et de la prise en charge globale des patientes.

\subsubsection{Connaissance par les prestataires de la période fécondité}

La connaissance de la fécondité dans le post abortum est un élément important surtout pour éviter les récidive dans un court délai. Aussi, l'effet de la formation sur la connaissance des prestataires sur le retour de la fécondité des patientes du post abortum est perceptible : en phase de pré-intervention, $29 \%$ savaient qu'une femme ayant avorté court le risque de devenir enceinte dans les deux semaines suivant l'avortement en cas de rapport sexuel non protégé tandis que dans la seconde phase, $70,7 \%$ le savent. Cette information est capitale pour convaincre les patientes et justifier la prescription de la contraception post avortement.

\subsection{LES COUTS DES PRESTATIONS DE SERVICES}

\subsubsection{Coûts supportés par la patiente}

Plusieurs études menées ailleurs ont relevé des coûts de soins élevés dans la prise en charge des soins du post abortum par dilatation curetage.

\subsubsection{Coûts des soins}

Les coûts moyens des ordonnances (soins) supportés par la patiente variaient de $1.875 \mathrm{~F}$ CFA à $75.775 \mathrm{~F} \mathrm{CFA}$ avec une moyenne de 12.249 F CFA. Ces frais ne prennent pas en compte les coûts des produits anesthésiques, des 
prestations de soins, des produits anticonceptionnels et ceux lié à leur séjour hospitalier.

\section{- Selon la technique d'évacuation utérine}

Il existe une différence très significative $(p<0,001)$ des coûts des soins selon la technique d'évacuation utérine utilisée.

- Les coûts moyens supportés par la patiente dans les cas de dilatation curetage (20.106 F CFA) sont deux fois plus élevées qu'en cas d'aspiration manuelle intra utérine (8.546 F CFA). Cette différence se justifie essentiellement par la frais des solutés et les autres produits nécessaires à la conduite de l'anesthésie ambulatoire réalisée lors de la dilatation curetage. La voie veineuse dite «de sécurité " permet d'administrer au bloc opératoire les drogues et certains produits de réanimation.

- Les coûts moyens du curage digital sont les plus faibles par rapport aux deux autres techniques du fait que cette procédure est utilisée en urgence, sans prise de voie veineuse ni antalgique particulier administré. Par ailleurs, cette technique a été abandonnée en seconde phase d'étude au profil de l'aspiration manuelle intra utérine qui a révélé au cours de la formation des utilisateurs la présence de débris ovulaires après la manœuvre.

\section{- Selon le type d'avortement}

Il apparaît une différence très significative au niveau des frais de soins, selon le type d'avortement. En effet, le coût moyen des frais d'ordonnance dans les cas d'avortement provoqué est de 14.126 F CFA alors qu'il n'est que de 9.765 F CFA dans les d'avortements spontanés. Ceci s'expliquerait surtout par la symptomatologie plutôt infectieuse des avortements provoqués, qui contraint à une double et même une triple association d'antibiotiques et d'anti-parasitaires de la part des prestataires.

\subsubsection{Les coûts de l'hospitalisation}

Les frais moyens d'hospitalisation ont été plus élevés dans les cas d'aspiration manuelle intra utérine (7.515 F CFA) qu'en cas de dilatation curetage (6.190 F CFA) ou en cas de curage digital (2.978 F CFA). Cette disparité des coûts pourrait se justifier par le circuit de la patiente et le temps d'attente avant la procédure d'évacuation des débris ovulaires.

- Les patientes ayant bénéficié du curage digital sont immédiatement libérées lorsque leur état clinique le permet. Ce qui rend brève leur durée de séjour hospitalier.

- La procédure de dilatation curetage est généralement conduite dans les 24 heures (sauf en cas d'indication de curetage hémostatique); ce qui rallonge le séjour des patientes.

- A l'introduction de l'aspiration manuelle intra utérine dans la prise en charge des patientes du post abortum, la durée de séjour hospitalier des patientes s'est raccourcie avec cette approche de prise en charge. 
- Au niveau du site de Bobo Dioulasso où l'unité de planification familiale est indépendante de l'unité de soins post abortum, la durée de séjour des patientes est plus élevée qu'à Ouagadougou.

\subsubsection{Coûts supportés par l'institution}

Dans le contexte du Burkina Faso, notre étude a retenu comme composantes des coûts liés à la prise en charge :

Les frais de soins, Les coûts liés à l'utilisation des drogues au cours des procédures d'anesthésie supporté par l'institution et, Les coûts liés à l'hospitalisation.

Les frais des soins supportés par l'Institution se résume dans notre étude aux produits anesthésiques qui ne sont pas l'objet de prescription.

Le coût moyen lié au contrôle de la douleur par anesthésie ambulatoire dans les cas de dilatation curetage est de 4.835 F CFA.

$\mathrm{Au}$ cours de l'utilisation de l'aspiration manuelle intra utérine, seulement 27,3\% des patientes ont bénéficiés d'une anesthésie loco-régionale à la Lidocaïne. Les frais des produits ont été supportés par les patientes.

La procédure de curage digital n'a pas engager de frais de soins médicamenteux dans notre échantillon d'étude.

Les coûts liés à l'utilisation des ressources matérielles et humaines sont difficiles à estimer. En effet, l'équipement hospitalier (instruments et mobilier chirurgical) a été acquis soit par dons (coopération internationale) ou acheté par le niveau central (ministère de la santé). Ce matériel dont les prix sont difficilement appréciables, ne peuvent bénéficier d'une cotation en taux d'amortissement leur attribuant de ce fait une valeur monétaire vraie.

De plus, les prestations de soins après avortement incomplet sont données dans un ensemble de prestations intégrées, le plus souvent avec l'appui des stagiaires internés de médecine et des internes. Les coûts liés à l'utilisation des ressources humaines ont donc été occultés du fait du manque d'objectivité dans les résultats de la collecte. Néanmoins, le temps de contact moyen selon la technique d'évacuation utérine utilisée est très variable :

- Dans les cas de curage digital, le temps moyen de contact est de 5 minutes pour une sage-femme ;

- Dans le cas de dilatation curetage, les ressources humaines utilisées sont plus nombreuses avec un temps de contact moyen variant suivant le grade du prestataire. Il est de

- 15 minutes pour le médecin (ou le stagiaire)

- 9 minutes pour la sage-femme

- 2 fois 21 minutes pour les aides opérateurs et anesthésistes

- 7 minutes pour le brancardier chargé de faire rentrer la patientes au bloc opératoire et de la sortir.

Ce qui donne un temps de contact moyen d'une (1) heure 13 minutes. 
- Pour l'aspiration manuelle intra utérine, les ressources humaines sont plus limitées par rapport à la dilatation curetage.

- 7 minutes en moyenne pour le médecin (ou le stagiaire)

- 16 minutes pour la sage-femme, soit un total de 23 minutes pour le temps de contact.

En somme, l'utilisation de l'AMIU réduit de 34,2\% les coûts supportés par la patiente et de $68,5 \%$ le temps de contact prestataires - patientes par rapport à l'utilisation de la dilatation curetage.

\subsection{LA QUALITE DES SOINS : OPINIONS DES PATIENTES}

Près du quart des suggestions des patientes est orienté vers l'amélioration des compétences (efficacité - efficience) des prestataires. Cela pourrait s'expliquer par l'insuffisance de communication entre prestataires et patientes. Cependant, les suggestions pour l'amélioration de la qualité de l'accueil des prestataires était deux fois moins évoquées au cours de la seconde phase, qu'en première.

Jusqu'à 43,2 \% des suggestions vont dans l'amélioration des états d'âme du personnel de santé. "Je ne suis pas mariée et j'ai déjà un enfant, vous comprenez que je ne voulais pas garder cette grossesse si mon partenaire refuse la paternité", la patience d'écoute (30,3\%), la compréhension des mobiles (13,9\%) ayant motivé l'interruption volontaire de grossesse, permettrons aux agents de santé d'être plus tolérants $(8,1 \%)$.

Quant aux propositions pour améliorer la qualité des services liée à l'institution; les suggestions des patientes sont diverses et pratiquement les mêmes aux cours des deux phases de l'étude.

- Par rapport aux coûts des ordonnances et des prestations de soins : 59,3\% des patientes estiment que l'établissement sanitaire doit mettre à la disposition des malades des médicaments et produits d'urgence. Il faut noter que les frais d'examens $(9,5 \%)$, d'hospitalisation $(2,2 \%)$ et de prestations de soins $(3,5 \%)$ sont moins évoquées. La notion "d'urgence" a toujours été difficile à discerner par un malade: en général, pour chaque malade admis dans un service hospitalier, son état est jugé grave par lui-même et son entourage.

- Enfin, pour rendre le séjour agréable, le service hospitalier doit porter des améliorations en mettant en place un service d'accueil et d'information $(38,3$ $\%$ ) et en prenant en charge la désinfection (14,7\%) des chambres et des toilettes, la distribution gratuite des repas (1\%), et aussi l'augmentation des capacités d'accueil $(16,0 \%)$ dans le respect de l'intimité des patientes $(4,2 \%)$. 


\subsection{CONNAISSANCE DES DISPOSITIONS JURIDIQUES ET REGLEMENTAIRES / OPINIONS SUR LA LEGISLATION EN MATIERE D'AVORTEMENT}

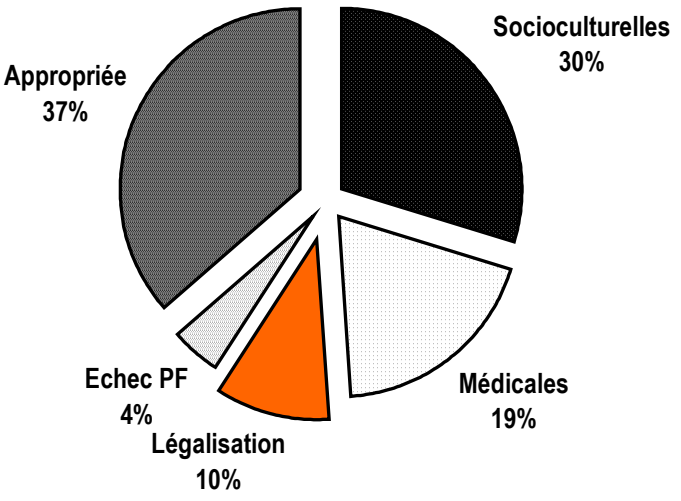

Fig 11 : Points de vue des patientes sur la Loi sur l'avortement provoqué au Burkina Faso
Au Burkina Faso, la Loi sur l'avortement ne l'autorise que dans les cas thérapeutiques dans le seul but de sauver la vie de la mère menacée par la poursuite de la grossesse ou encore lorsqu'une malformation fotale est prouvée. Elle a été élargie aux cas d'inceste ou de viol prouvé par une autorité compétente. En somme, l'avortement provoqué est interdit. Cette interdiction

est connue par 70,7 \% des patientes qui la trouvent dans 33,5\% des cas restrictive et libérale dans $37,0 \%$. Le contexte dans lequel l'avortement thérapeutique est autorisé n'est connu que par $55,2 \%$ des patientes dans son ancienne formulation et un peu moins de $25,0 \%$ dans sa nouvelle formulation.

Seulement, 10,0 \% des patientes propose la libéralisation de l'avortement autorisant ainsi le couple ou à la femme d'avoir des prestations de soins abortifs à la demande. 


\section{CONCLUSION}

Première étude pour l'introduction de la prise en charge intégrée des avortements dans un pays d'Afrique francophone associant les soins curatifs, la technique d'AMIU aux conseils en planification familiale, ce projet revêt beaucoup d'intérêts. Il a permis une amélioration de la qualité des prestations par la formation des agents de santé en prévention des infections et la mise à jour de leurs compétences en technologie contraceptive. Elle a favorisé une réorganisation fonctionnelle des services de soins et surtout l'adhésion des responsables des services de gynécologie et obstétrique à la nouvelle approche.

Au regard de l'attitude négative habituelle des prestataires à l'égard des avortements, la présente recherche opérationnelle a créé des changements de comportement positifs tant dans les relations interpersonnelles que dans l'adhésion à l'introduction des SPA / PF dans la prise en charge des avortements incomplets, attribuables en grande partie aux formations en counseling et en planification familiale post abortum.

Cette nouvelle approche à démontré comme avantages :

- Un accroissement de la qualité des services,

- Une réduction des coûts des prestations pour les patientes,

- Une réduction de la charge en travail des prestataires,

- Une augmentation du taux d'acceptabilité de la PF,

Ces résultats qui entrent en droite ligne des préoccupations de la CIPD en 1994, nous exhortent à rechercher de stratégies pour la pérennisation de cette approche dans nos deux hôpitaux nationaux et à son extension au niveau des centres médicaux de prise en charge des complications des avortements.

Ces stratégies devront privilégier le respect de la législation en cours et la politique de décentralisation en cours de réalisation pour les formations sanitaires. 


\section{RECOMMANDATIONS}

\subsection{Par rapport au programme SPA}

- L'élargissement du programme de soins post abortum / PF aux hôpitaux régionaux du Burkina Faso avec inclusion des coûts des équipements liés au programme dans les budgets des hôpitaux.

- Intégration des activités des services de SPA dans les autres activités de santé des unités techniques.

- Validation du modèle de service SPA et les normes et standard pour l'extension du programme.

- La formation des prestataires de soins doit être continue et s'adresser aussi à la base en mettant l'accent sur la qualité des services (relations interpersonnelles).

- Les hôpitaux site d'étude doivent être des centres de référence pour la formation des prestataires et des sites d'étude pour l'appréciation de nouveaux aspects liés à la santé de la reproduction.

\subsection{Par rapport aux prestations}

- L'utilisation de l'aspiration manuelle intra utérine comme technique d'évacuation des débris ovulaires est idéal pour la prise en charge des patientes admises dans les services hospitaliers pour avortement incomplet non compliqué,.

Cette technique ne doit pas faire éclipser les autres techniques.

- Il est plus indique d'intégrer les services de soins post abortum aux services de $\mathrm{PF}$ avec renforcement en personnel qualifié.

Le contrôle de la douleur : La préparation psychologique de la patiente et L'utilisation d'une anesthésie loco-régionale doit dépendre de la volonté de la patiente qui seule peut juger son état de perception de la douleur.

La participation des hommes lors du counseling en PF pourrait amener le couple à faire le choix de la méthode contraceptive qui lui correspond au mieux. 


\section{BIBLIOGRAPHIE}

1. A.D.P. IV République 1 ère Législature. Loi N²3/94/A.D.P. portant sur le code de la santé publique. Burkina Faso.

2. Abdel-Tawab N., Huntington D. et coll. Effets of husband involvement on post abortion patientes recovery and use of contraception in Egypte. January 19-21, 1998. Population Council, New York

3. Bazié A. J., Dao B. et coll. Les avortements provoqués clandestins à la maternité du Centre Hospitalier National Sanou Sourou (à propos de 158 cas). 1996.

4. CE.FO.REP. Dakar. Introduction des soins obstétricaux d'urgence et de la planification familiale pour les patientes présentant des complications liées à un avortement. Septembre 1998. Population Council, New York.

5. Coeytaux Francine M., Ann H. Leonard and Carolyn M. Bloomer "Abortion", in The Health of Women, A Global Perspective, Marge Koblinsky, Judith and Jill Gay editors, Westerview Press, 1993.

6. Coeytaux Francine, "Abortion, Sexually Transmitted Diseases and Infertility : Reproductive Health Problems Family Planning Programs Can No longer Ignore" Paper presented at the 1990 NCIH Conference.

7. Ghosh A., Koné B., Lankoandé J., Tapsoba P. Introducing improved post abortion care into maternity service in Burkina Faso, Junuary 19-21, Population Council, New York.

8. Greenslade, F., H. McKay, M. Wolf \& K. McLaurin (1994) "Post-Abortion Care: A women's health initiative to combat unsafe abortion", Advances in Abortion Care, 4,1.

9. Gué M.J., Yaméogo B. et coll. Enquête CAP sur la santé de la reproduction dans 10 villages de la province du Sanmatenga. Plan de Parrainage International. 1996.

10. Huntington D. et coll. A descriptive study of the post abortion cure load in Egyptian Hospital. Junuary 19-21, 1997. Population Council, New York.

11. I.P.P.F. Conférence de Maurice : Les avortements à risque et la planification familiale post abortum en Afrique. 1994, Ile Maurice.

12. Institut National de la Statistique et de la Démographie. Enquête de démographique et de santé 1993. Burkina Faso. 
13. Johnson, B., J. Benson, J. Bradley \& A. Ordonez (1993) "Costs and resource utilization for the treatment of incomplete abortion Kenya and Mexico" Social Science and Medicine, 36,11:1443-1453.

14. JuanDiaz et Col, PC-1998

15. Kim T., Benson J., Stein K. Comparing the cost of post abortion cure in Africa and latina America. The data post abortion cure projet. January 19-21, 1998, Population Council, New York.

16. Kizza A.P.M. and Roggo K.O. Assessement of the manual vacuum aspiration in the management of incomplete abortion. East African médical Journal, Volune $67, \mathrm{~N}^{\circ} 11$, November $1990 ; 812-822$.

17. Langer A. and coll. Improving post abortion care in a public hospital in Oaxaca, Mexico. January 19-21, 1997. Population council, New York.

18. M. Abernathy et al (1993) A Guide to Assessing Resource Use for the Treatment of Incomplete Abortion, Ipas, Carrboro, USA.

19. McLaurin, Katie E., Horde, Charlotte and Merrill Wolf "Health Systems, Role in Abortion Care : The Need for Brow Active Approach" Advances in Abortion IPAS Carrboro North Carolina, 1991.

20. Nahla Abdel-Tawab, PC-1998

21. World Health Organization "Protocol for Hospital Based Descriptive Studies for Mortality, Morbidity Related to Induced Abortion" WHO Project Nº. 86912, Task Force on Safety and Efficacy of Fertility Regulating Methods, revised edition of August 14th, 1987. 